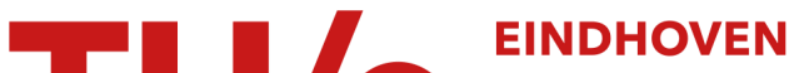 \\ UNIVERSITY OF \\ TECHNOLOGY
}

\section{Integrating autonomous delivery service into a passenger transportation system}

Citation for published version (APA):

Mourad, A., Puchinger, J., \& van Woensel, T. (2021). Integrating autonomous delivery service into a passenger transportation system. International Journal of Production Research, 59(7), 2116-2139.

https://doi.org/10.1080/00207543.2020.1746850

\section{Document license:}

TAVERNE

DOI:

10.1080/00207543.2020.1746850

Document status and date:

Published: 01/01/2021

\section{Document Version:}

Publisher's PDF, also known as Version of Record (includes final page, issue and volume numbers)

\section{Please check the document version of this publication:}

- A submitted manuscript is the version of the article upon submission and before peer-review. There can be important differences between the submitted version and the official published version of record. People interested in the research are advised to contact the author for the final version of the publication, or visit the $\mathrm{DOI}$ to the publisher's website.

- The final author version and the galley proof are versions of the publication after peer review.

- The final published version features the final layout of the paper including the volume, issue and page numbers.

Link to publication

\section{General rights}

Copyright and moral rights for the publications made accessible in the public portal are retained by the authors and/or other copyright owners and it is a condition of accessing publications that users recognise and abide by the legal requirements associated with these rights.

- Users may download and print one copy of any publication from the public portal for the purpose of private study or research.

- You may not further distribute the material or use it for any profit-making activity or commercial gain

- You may freely distribute the URL identifying the publication in the public portal.

If the publication is distributed under the terms of Article $25 \mathrm{fa}$ of the Dutch Copyright Act, indicated by the "Taverne" license above, please follow below link for the End User Agreement:

www.tue.nl/taverne

Take down policy

If you believe that this document breaches copyright please contact us at:

openaccess@tue.nl

providing details and we will investigate your claim. 


\section{International Journal of Production Research}

\section{Integrating autonomous delivery service into a passenger transportation system}

\section{Abood Mourad, Jakob Puchinger \& Tom Van Woensel}

To cite this article: Abood Mourad, Jakob Puchinger \& Tom Van Woensel (2021) Integrating autonomous delivery service into a passenger transportation system, International Journal of Production Research, 59:7, 2116-2139, DOI: 10.1080/00207543.2020.1746850

To link to this article: https://doi.org/10.1080/00207543.2020.1746850

\section{Published online: 06 Apr 2020.}

Submit your article to this journal

Llll Article views: 574

Q View related articles $₫$

View Crossmark data $₫$

4

Citing articles: 4 View citing articles $ک$ 


\title{
Integrating autonomous delivery service into a passenger transportation system
}

\author{
Abood Mourad $\mathbb{1}^{\mathrm{a}, \mathrm{b} *}$, Jakob Puchinger ${ }^{\mathrm{a}, \mathrm{b}}$ and Tom Van Woensel ${ }^{\mathrm{c}}$ \\ ${ }^{a}$ Laboratoire Genie Industriel, CentraleSupélec, Université Paris-Saclay, Gif-sur-Yvette, France; ${ }^{b}$ Institut de Recherche Technologique \\ SystemX, Palaiseau, France; ${ }^{c}$ School of Industrial Engineering, Eindhoven University of Technology, Eindhoven, The Netherlands
}

(Received 22 May 2019; accepted 5 March 2020)

\begin{abstract}
The increasing demand for goods, especially in urban areas, together with the technological advances are creating both opportunities and challenges for planning urban freight systems. One of these promising opportunities is to use the underused assets in people-based systems to transport goods. In this paper, we consider an integrated system in which a set of freight requests needs to be delivered using a fleet of grounded, and autonomous, pickup and delivery (PD) robots where a public transportation service (referred to as scheduled line (SL)) can be used as part of PD robot's journey. Passengers and PD robots (carrying freight) share the available capacity on SLs where passengers are prioritised, and their transport demand is stochastic. Thus the number of available places for PD robots is only revealed upon shuttle arrival to the corresponding SL station. We first formulate this problem as a Pickup and Delivery Problem with Time Windows and Scheduled Lines (PDPTW-SL). We then introduce a sample average approximation (SAA) method along with an Adaptive Large Neighbourhood Search (ALNS) algorithm for solving the stochastic optimization problem. Finally, we present an extensive computational study, analyse its results and give some directions for future research.
\end{abstract}

Keywords: pickup and delivery; scheduled lines; stochastic demand; ALNS; sample average

\section{Introduction}

The demand for freight transportation basically results from the need of transporting goods from producers to consumers who are geographically apart. In general, this transportation process consists of picking up products at their producer locations (pre-haul), transporting them (long-haul) and delivering them to final consumers (end-haul) at the right time and place and at low costs (Steadieseifi et al. 2014). The increasing demand for goods in urban areas, together with the emerging information and technological advances are creating both opportunities and challenges for planning urban freight systems (Savelsbergh and Van Woensel 2016). One of these opportunities is to represent supply chains as networks of physical objects that are digitally connected, referred to as Internet-of-Things (IoT), in order to facilitate planning and control of their processes and enable tracking and information sharing (Ben-Daya, Hassini, and Bahroun 2019). In addition, the latest development in social media and its applications provide new features for supply chain planning, such as understanding customers' preferences and forecasting their demands, building trust and customer relationship and so on (Huang, Potter, and Eyers 2020). These features require new decision-making models yielding efficient planning of production and transportation activities (Rossit, Tohmé, and Frutos 2019). In this paper, we consider one of these promising opportunities which is to use the low-utilised people transport systems (e.g. off-peak hours of urban rail, buses or private car trips) to also transport goods. A successful integration of these transportation streams can enhance the service quality of their existing transportation systems as well as their system-wide gains. For example, spare capacity in public transport systems can be used for retail store replenishment (Trentini et al. 2015) or a taxi can deliver freight when transporting a passenger or during idle time (Li et al. 2014).

In such combined systems, we have a set of passengers and parcels that need to be transported simultaneously from their origins to their destinations. This combination can lead to minimising vehicle miles travelled, traffic congestion and pollution levels in urban areas. It can also yield some travel cost reductions for passengers. However, such a system must ensure that the transportation of goods does not disturb passenger trips. In other words, a passenger would accept only small deviations and short extra times when transporting some parcels in the same trip (i.e. trip times that exceed passenger usual route times significantly might not be acceptable).

\footnotetext{
*Corresponding author. Email: mouradabood90@gmail.com
} 
In this paper, we consider an integrated system in which a set of freight requests needs to be transported from their origins to their destinations. We use a fleet of grounded and autonomous pickup and delivery (PD) robots where a public transportation service (e.g. a set of shuttles, referred to as scheduled line $(S L)$ ) can be used as part of PD robot's journey. ${ }^{1}$ Most research considers that passengers and goods are transported separately. However, we consider that passengers and PD robots (carrying goods) share the same capacity. This implies that a freight request can be served in one of two ways: (1) a direct delivery (where only a PD robot is used) or (2) transferred through SLs (where both PD robots and SLs are used). Therefore, a parcel might be picked up at its origin location by a PD robot, transported through the scheduled line with passengers, and delivered to its final destination by the PD robot. In order to guarantee an acceptable service quality for passengers, they are assumed to have a higher priority to use SL service. In other words, PD robots are only able to use SLs when there are free places available (i.e. not used by any passengers).

A similar system is considered by Ghilas, Demir, and Van Woensel (2016b) where a scheduled line service is used along with a fleet of heterogeneous vehicles to serve a set of freight requests. In their system, the exact quantities demanded by each customer are only learned upon vehicles' arrival at the corresponding pickup locations. Unlike their problem settings, we consider that freight quantities are known in advance. In addition, we consider that passengers demand for transportation is only learned upon the shuttles' arrival to each SL station. Since passengers and PD robots share the same capacity on SLs, the number of available places for PD robots at each SL departure is thus stochastic. Depending on the actual passengers transport demand, there are two possible violation outcomes: (i) the PD robot is not able to take the next SL departure due to the high passenger demand at the corresponding station and (ii) the PD robot needs to get off the SL at an intermediate station, where passengers demand is high, in order to give its place to a passenger. When these route failures occur, a number of recourse actions are needed in order to recover feasibility. Applying these recourse actions might lead to extra handling and transportation costs compared to their original routes.

The key contributions of this paper are as follows. First, we model the proposed pickup and delivery problem as a twostage stochastic problem. The first stage consists of defining routes for PD robots carrying freight requests. These routes are evaluated over a set of scenarios and their associated recourse costs are calculated in the second stage. The overall objective is to minimise the overall transportation costs (i.e. the sum of the first-stage routing costs and the second-stage recourse costs). Second, we propose a sample average approximation (SAA) method along with an Adaptive Large Neighbourhood Search (ALNS) algorithm for solving the stochastic optimization problem. Finally, we provide a computational study to quantify the impact of passengers demand realization on such combined systems. This is achieved by comparing the solutions obtained when deterministic and stochastic versions of the problem are solved. While the potential benefits of integrating parcel deliveries to SL service were extensively studied in Ghilas, Cordeau, and Demir (2016), in this paper we aim at studying the impacts of stochastic passenger demands on this system with different SL frequencies and capacities.

This paper is organised as follows. In Section 2, we provide an overview of related literature. In Section 3, we describe the problem, provide a mathematical formulation for it, and introduce an algorithm to evaluate its solutions and calculate their recourse costs. The proposed solution method is detailed in Section 4. In Section 5, we present the computational study and analyse its results. Finally, in Section 6, the key findings are summarised and directions for future research are suggested.

\section{Background}

An increasing amount of research is being directed recently towards studying and developing new transportation systems that integrate passenger and freight flows. These systems can be classified into single-tiered and two-tiered systems (Mourad, Puchinger, and Chu 2019). In single-tiered systems, a set of vehicles transport passengers and goods to their destinations while taking into account some considerations (e.g. request time windows, vehicle capacity, etc.). On the other hand, passenger and freight flows are combined in two-tiered systems thanks to the contribution of a first-tier (e.g. a public mass-transport line), and a second-tier (e.g. a fleet of vehicles) that performs the last-mile deliveries to customers (see Dolati Neghabadi, Evrard Samuel, and Espinouse 2019 for a recent review on city logistics). In both cases, evaluating the performance of these systems is essential for achieving sustainability and ensuring a balance among the different economic, environmental and social aspects (Jamshidi et al. 2019). For this purpose, Pathak, Thakur, and Rahman (2019) introduced a comprehensive framework to assess the sustainability performance of such systems in order to help decision-makers in selecting the most sustainable model for their transportation systems.

Regarding single-tiered systems, Li et al. (2014) introduced the Share-a-Ride Problem (SARP) in which passenger and freight requests are transported using a fleet of taxis driving around in a city. As passenger requests are given a higher priority, some parcels are delivered during taxi trips in case this delivery does not affect the passengers significantly. For solving the SARP, an MILP formulation, that extends the classical Dial-a-Ride problem, along with an ALNS method were 
proposed (see also Li et al. 2016a). Their results demonstrated the benefits of such combination in terms of transportation costs and travelled distances. These benefits were observed by comparing results to those where passenger and freight requests are served separately. In another study, Arslan et al. (2016) presented an event-based rolling horizon framework that dynamically assigns parcel deliveries to self-employed drivers who are willing to earn some extra money by making deliveries on their way to home or work. In addition, the authors proposed a heuristic recursive algorithm for solving the routing subproblem. Their results demonstrated that this integrated delivery can potentially reduce last-mile delivery costs as well as the system-wide vehicle miles. Archetti, Savelsbergh, and Speranza (2016) considered a similar single-tiered model where a set of occasional drivers is used to supplement the service provided by delivery vehicles and dedicated drivers. Occasional drivers are those willing to make a single delivery using their own vehicle. The authors modelled this problem as a Vehicle Routing Problem (VRP) with occasional drivers and proposed a heuristic approach that uses variable neighbourhood and tabu search strategies for solving it. Their results showed that introducing more occasional drivers to the system can decrease the total transportation cost and the number of dedicated drivers required. Moreover, Wang et al. (2016) presented a single-tiered model where last-mile deliveries are performed by a large-pool, a crowd, of citizen workers. The proposed model was formulated as a network min-cost flow problem and solved using an iterative pruning technique. Furthermore, Dayarian and Savelsbergh (2017) suggested that potential customers can express their interest to participate in making deliveries on their way home. The authors proposed a tabu search heuristic method for generating vehicle routes.

On the other hand, some recent studies have focused on studying two-tiered systems. These systems include transporting freight alongside passengers on rail transit and bus lines, or attaching cargo trailers to transit vehicles for example (see Cochrane et al. 2017 for a recent review with many real-life examples). Fatnassi, Chaouachi, and Klibi (2015) introduced an integrated system where passengers and goods are transported to intermediate points using a first tier (train, bus or truck line), and then delivered using a fleet of electric vehicles (second tier). The authors proposed a forward periodic-optimization approach which showed that the proposed system can achieve a potential gain in terms of service time and energy consumption. Another study, by Masson et al. (2017), considered a combined system that uses the available capacity in a passenger bus line to transport parcels to specific bus stations where a fleet of low-emission freighters delivers them to final customers. The paper formulated the system as a Vehicle Routing Problem with transfers and proposed an ALNS-based heuristic to solve it (see also Trentini et al. 2015). Similarly, Ghilas, Cordeau, and Demir (2016) introduced a two-tiered system where parcels are delivered by a fleet of vehicles such that a part of the delivery process is carried out on a scheduled line of public transport. The paper modelled this integrated system as Pickup and Delivery Problem with Time Windows and Scheduled Lines (PDPTW-SL) and introduced an ALNS-based algorithm for solving it. Their results showed that an average cost savings of $10 \%$ can be achieved thanks to the use of the scheduled line compared to a pure-freight delivery system. Moreover, Kafle, Zou, and Lin (2017) suggested that parcels can be transported to intermediate points using a set of carrier trucks, and then delivered by a set of potential cyclists and pedestrians who are living in the same neighbourhood. The authors proposed a tabu search algorithm for solving the associated optimization problem. Their results demonstrated that the use of potential cyclists and pedestrians can reduce the operational costs by $9.25 \%$ compared to a truck-based delivery system. Studying a freight-rail transport system, Behiri, Belmokhtar-Berraf, and Chu (2018) introduced a model where freights are brought to rail stations by trucks at different time periods in a day, before being shipped through railways. To operate this system, the authors introduced a dispatching rule-based heuristic approach and highlighted the benefits of the proposed model. Furthermore, Ozturk and Patrick (2018) considered a system where the same railway is shared between passenger trains and freight trains. After introducing a mixed integer linear programming model for the system, the study presented an approximation algorithm and a pseudo-polynomial dynamic programming approach to solve it and evaluated their performances through a numerical study.

As for studying uncertainty in such combined systems, Li et al. (2016b) extended the SARP, introduced earlier, by considering two stochastic variants. The first variant considered the travel times to be stochastic while the second considered stochastic delivery locations. For solving both variants, a two-stage stochastic programming model with recourse is used with the ALNS heuristic and a scenario generator. Through an extensive experimental study on both stochastic models, this paper concluded that the stochastic travel times have a more noticeable effect on the SARP than the stochastic delivery locations. In addition, Ghilas, Demir, and Van Woensel (2016b) extended their two-tiered model by considering stochastic demand quantities of freight requests which are only revealed upon the vehicle's arrival to their pickup locations. A scenario-based sample average approximation approach was introduced in order to consider this uncertainty (see Ritzinger, Puchinger, and Hartl 2016 for a review on stochastic VRPs and their solution methods). After reviewing the related literature, we provide a detailed description of the considered problem along with the method used to solve it in the following sections. 


\section{Problem description}

Consider a set of shuttles that operate on a scheduled line (SL) service in both directions. This service consists of a set of physical transfer nodes (i.e. stations) $\mathcal{S}$, where passengers take shuttles as part of their trip to their final destinations, and a set of physical scheduled lines $\mathcal{E}$ linking different transfer nodes. Between every pair of transfer nodes $i, j \in \mathcal{S}$, there are two scheduled lines with opposite directions $(i, j),(j, i) \in \mathcal{E}$. Shuttles move through the scheduled line in fixed routes. Every shuttle moving through scheduled line $(i, j)$ has a capacity $Q_{i j}$, indicating the number of available places, and a schedule $\mathcal{K}^{i j}$, indicating its departure times at origin transfer node $i$ (denoted by $p_{i j}^{w}$, e.g. the second departure from $s_{1}$ to $s_{2}$ is $p_{s_{1}, s_{2}}^{1}=60$ time units). Moreover, shipping one unit of package on scheduled line $(i, j)$ is associated with a cost $\eta_{i j}$ per unit. In addition, a fleet of autonomous, pickup and delivery (PD) robots are located at transfer nodes. Each PD robot $v \in \mathcal{V}$ is assigned to a depot (i.e. transfer node) $o_{r} \in \mathcal{S}$ and has a capacity $Q_{v}$ and a maximum service distance $\delta_{v}$ indicating the maximum distance it can go from a transfer node to a request pickup or destination location. ${ }^{2}$ Each PD robot is associated with a routing cost per time unit $\theta_{v}$.

In addition, a set of freight requests need to be transported to their final destinations using the fleet of PD robots. Each request is associated with an origin $r \in \mathcal{P}$ and a destination $r+n \in \mathcal{D}$ (where $n=|\mathcal{P}|$ is the number of requests), indicating where it should be picked up and to where it should be delivered. In addition, request $r$ is associated with two time windows, a pickup time window $\left[e_{r}, l_{r}\right]$ and a delivery time window $\left[e_{r+n}, l_{r+n}\right]$, and a demand quantity $u_{r}$. Pickup and delivery time windows indicate when the request should be picked up by a PD robot and when it should be delivered to its final destination. Depending on the availability of vacant places in SLs, PD robots carrying freights may travel with passengers between different transfer nodes. A freight, carried by a PD robot, can thus be transported by a shuttle between two transfer nodes as part of its journey.

Indeed, allowing passengers and PD robots to travel simultaneously aims at using the spare capacity in shuttles especially that loading (and unloading) these robots into shuttles at transfer points come with relatively short service times. As a result, delivering a request to its final destination can be done in either direct or indirect way (see Figure 1). In a direct delivery, a request is picked up by a PD robot at its origin and delivered directly to its final destination without the use of the scheduled line (Figure 1a; request $a_{1}$ is picked up at its origin $o_{a_{1}}$ by a PD robot coming from transfer node $s_{2}$, and delivered to its final destination $d_{a_{1}}$ before the PD robot returns to transfer node $s_{3}$ ).

It is important to mention that a direct delivery is only feasible if the distance between the transfer node and request origin/destination, and between request origin and destination locations is less than the maximum distance the robot can travel. In Figure 1(b), if the distance between $o_{a_{1}}$ and $d_{a_{1}}$ is greater than the robot maximum service distance, a direct delivery cannot be performed and the SL service must be used. On the other hand, in an indirect delivery, a request may be collected by one PD robot, transferred through the scheduled line and delivered afterwards to its final destination by the same PD robot (Figure $1 \mathrm{~b}$, request $a_{1}$ is picked up at its origin $o_{a_{1}}$ by a PD robot, brought to transfer node $s_{2}$, transported through the scheduled line from $s_{2}$ to $s_{3}$ and finally delivered to its final destination $d_{a_{1}}$ by the PD robot).

Since passengers and PD robots are using SLs simultaneously in indirect deliveries, we assume that a passenger or a PD robot needs one place in a shuttle while passengers have higher priority to be transported. We also assume that PD robots cannot take over more than a fixed number of places in each shuttle (e.g. if the shuttle capacity is 10 places, PD robots can take over at most three places). We assume that each PD robot can serve only one freight request at a time. In other words,

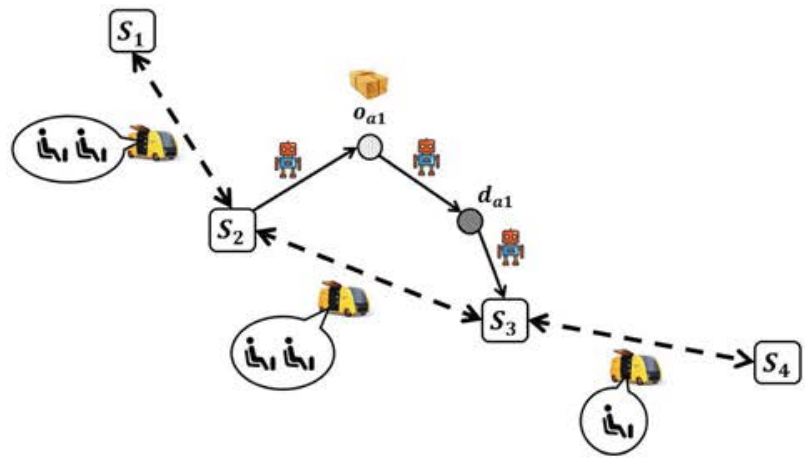

(a)

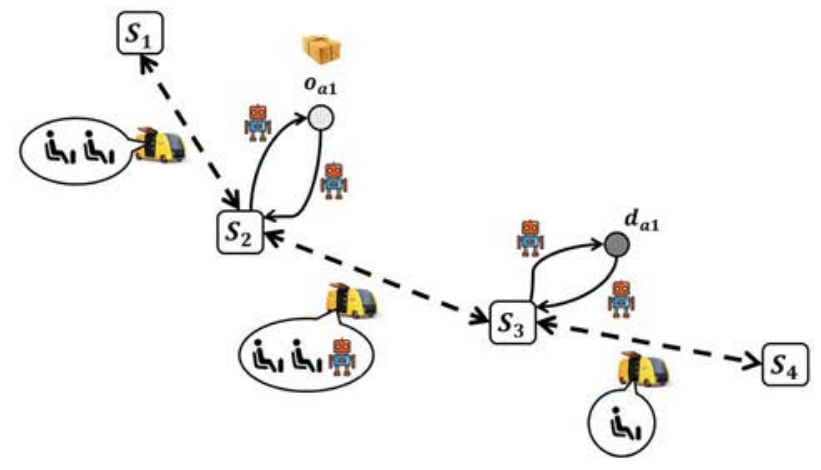

(b)

Figure 1. Request service modes: direct and indirect delivery: (a) direct delivery - PD robot and (b) indirect delivery - PD robot and SL. 


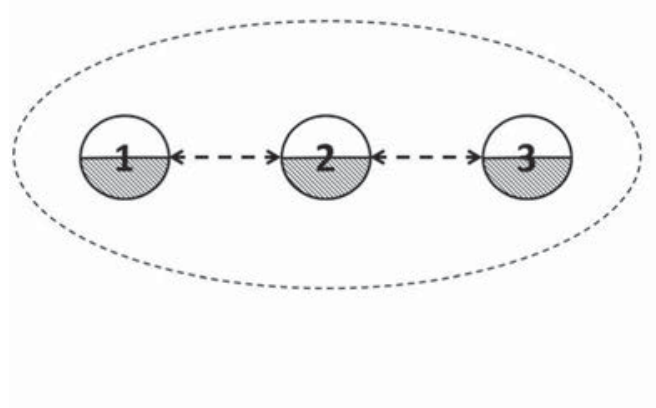

(a)

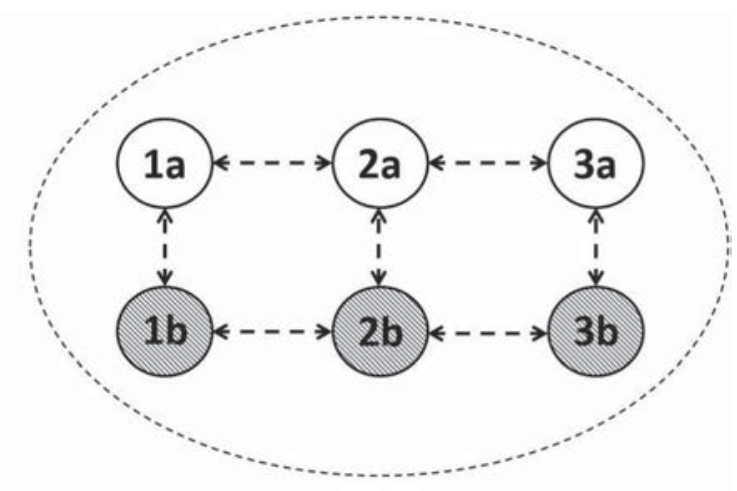

(b)

Figure 2. Scheduled line with four replicated transfer nodes: (a) physical scheduled line and (b) virtual scheduled line.

a PD robot can only pickup one request from its origin to a transfer point and deliver it from a transfer point to its final destination during one single trip. This assumption can be relaxed so as to consider more realistic settings in which a PD robot can perform multiple pickups and deliveries during a single trip.

Furthermore, the following set of assumptions is used throughout the paper:

- SLs are assumed to be homogeneous in terms of frequency and capacity.

- We assume that all the shuttles operating on SLs have the same capacity. Each shuttle is thus assumed to have a maximum number of places to transport both passengers and PD robots.

- We assume that a PD robot might return to a different station than the one it departed from (as it is the case in Figure 1a) after delivering its request (i.e. relocation operations are not considered).

- As PD robots are likely to be electric ones, a PD robot is assumed to be fully charged at each time it departs from a transfer node for picking up or delivering a request and that this charge is enough to perform its trip (recharging operations are not considered).

- It is also assumed that each PD robot has a storage compartment (where parcels are stored during the robot trip) and those compartments are assumed to be homogeneous.

- Regarding freight demands, it is assumed that the exact quantity and delivery time windows of each request are known beforehand.

- In addition, we assume that each demand unit corresponds to a package of a standardised small size so that it can fit in robot storage compartments (content, nature and weight of the package are disregarded).

- Finally, we assume that shuttles operate through dedicated lanes, and their travel times are thus known beforehand (i.e. no traffic-related delay can be encountered). We also assume that service times at transfer nodes are known beforehand and remain unchanged during the planning horizon. In other words, we fix the service time at each SL station and assume that it is enough for both passengers and PD robots to get in or out of shuttles.

Similar to Ghilas, Demir, and Van Woensel (2016a), each scheduled line is replicated in $n$ copies $(n=|\mathcal{P}|$ is the number of requests). Each replication is assigned to one request, and only that specific request can travel on the assigned scheduled line. These replications are used in order to reduce the number of decision variables in the model. Figure 2 illustrates an example in which we have three transfer nodes $\{1,2,3\}$, two physical scheduled lines (i.e. arcs $(1,2),(2,1),(2,3)$ and $(3,2))$ and two requests $\{a, b\}$. The set of all replicated scheduled lines is denoted by $\mathcal{F}$ (i.e. $\{(1 a, 2 a),(1 b, 2 b),(2 a, 3 a),(2 b, 3 b),(2 a, 1 a),(2 b, 1 b),(3 a, 2 a),(3 b, 2 b)\}$ in Figure 2(b)). Furthermore, the set of replicated SLs associated with request $r$ is given as $\mathcal{F}^{r}$ (e.g. in Figure $\left.2, \mathcal{F}^{a}=\{(1 a, 2 a),(2 a, 1 a),(2 a, 3 a),(3 a, 2 a)\}\right)$. In addition, the set of replicated SLs related to the replicated transfer node $t$ is given as $\mathcal{F}^{t}$ (e.g. $\mathcal{F}^{1 a}=\{(1 a, 2 a),(2 a, 1 a)\}$ ). Finally, $\mathcal{F}^{i j}$ includes all replicated SLs associated with a physical SL $(i, j) \in \mathcal{E}$ (e.g. $\mathcal{F}^{1,2}=\{(1 a, 2 a),(1 b, 2 b)\}$ and $\left.\mathcal{F}^{2,1}=\{(2 a, 1 a),(2 b, 1 b)\}\right)$ (Figure 3).

Furthermore, each transfer node in $\mathcal{S}$ is copied $n$ times. Hence, we denote the set of all replicated transfer nodes by $\mathcal{T}$ (i.e. $\mathcal{T}=\{1 a, 1 b, 2 a, 2 b, 3 a, 3 b\}$ in Figure $2 b$ ). In addition, we use $\psi^{t}, \forall t \in \mathcal{T}$ as the physical transfer node represented by the replicated transfer node $t$ (e.g. $\psi^{1 a}=\psi^{1 b}=1$ ).

The proposed pickup and delivery problem is defined on a digraph $\mathcal{G}=(\mathcal{N}, \mathcal{A})$ where $\mathcal{N}=\mathcal{P} \cup \mathcal{D} \cup \mathcal{T}$, represents the set of graph nodes (i.e. request origins, destinations and replicated transfer nodes), and $\mathcal{A} \equiv \mathcal{A}_{1} \cup \mathcal{A}_{2} \cup \mathcal{A}_{3}$ represents the 

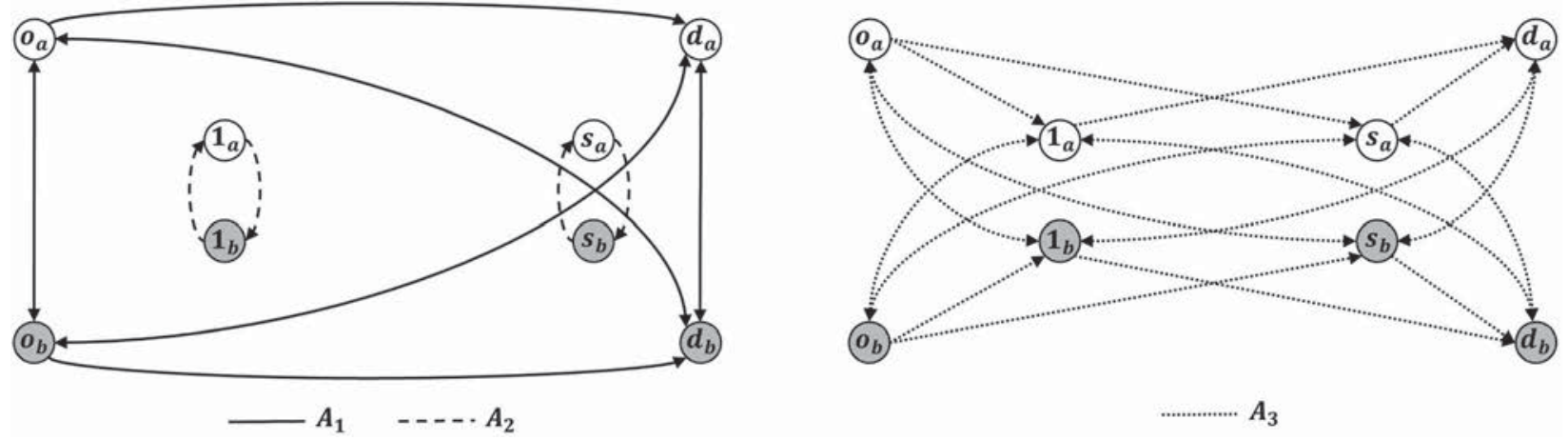

Figure 3. An example network with $s$ replicated nodes and two requests.

set of feasible arcs connecting different graph nodes, where subset $\mathcal{A}_{1}$ represents arcs linking request origin and destination nodes, subset $\mathcal{A}_{2}$ represents arcs linking replicated transfer nodes, and subset $\mathcal{A}_{3}$ links request origin and destination nodes to transfer nodes (3).

For modelling the problem, we introduce two binary variables; $x_{i j}^{v}$ equals to 1 if arc $(i, j)$ is used by PD robot $v$ and 0 otherwise, $\forall(i, j) \in \mathcal{A}, v \in \mathcal{V}$, and $q_{i j}^{v w}$ equals to 1 if replicated scheduled line $(i, j)$ is used by PD robot $v$ that departs from node $i$ at time $p_{i j}^{w}$ and 0 otherwise, $v \in \mathcal{V},(i, j) \in \mathcal{F}^{i} j, w \in \mathcal{K}^{i j}$. In addition, we introduce two timing variables; $\beta_{i}$ indicates the departure time of a PD robot from node $i \in \mathcal{N}$, and $\gamma_{i}^{v}$ which indicates the departure time of a PD robot $v \in \mathcal{V}$ from replicated transfer node $i \in \mathcal{T}$. $\beta_{i}$ is used to ensure the compatibility of departure times between two consecutive nodes, while $\gamma_{i}^{v}$ is used to synchronise the departure of PD robots with the departure of SLs at stations (notations and variables used in this paper are summarised in Table 1). We present a two-stage stochastic model in the following sections.

\subsection{The first-stage model}

$$
\operatorname{Min} \sum_{(i, j) \in \mathcal{A}} \sum_{v \in \mathcal{V}} \theta_{v} t_{i j} x_{i j}^{v}+E[Q(\Gamma, \xi, \eta)]
$$

subject to

Routing and flow constraints

$$
\begin{aligned}
& \sum_{i \in \mathcal{N}} \sum_{v \in \mathcal{V}} x_{i j}^{v}=1 \quad \forall j \in \mathcal{P} \cup \mathcal{D} \\
& \sum_{i \in \mathcal{N}} x_{o_{v}}^{v} \leq 1 \quad \forall v \in \mathcal{V} \\
& \sum_{i \in \mathcal{N}} \sum_{v \in \mathcal{V}} x_{i t}^{v} \leq 1 \quad \forall t \in \mathcal{T} \\
& \sum_{j \in \mathcal{N}} x_{i j}^{v}-\sum_{j \in \mathcal{N}} x_{j i}^{v}=0 \quad \forall i \in \mathcal{N}, \forall v \in \mathcal{V} \\
& \sum_{t \in \mathcal{T}} x_{i t}^{v}-\sum_{t \in \mathcal{T}} x_{t j}^{v}=0 \quad \forall v \in \mathcal{V}, \forall(i, j) \in \mathcal{P} \times \mathcal{D} \\
& t_{i j} x_{i j}^{v} \leq \delta_{v} \quad \forall i, j \in \mathcal{N}, \forall v \in \mathcal{V}
\end{aligned}
$$

Capacity constraints

$$
\sum_{i \in \mathcal{T}} \sum_{v \in \mathcal{V}} u_{r} x_{i r}^{v} \leq Q_{v} \quad \forall r \in \mathcal{P}
$$


Table 1. Notations and variables.

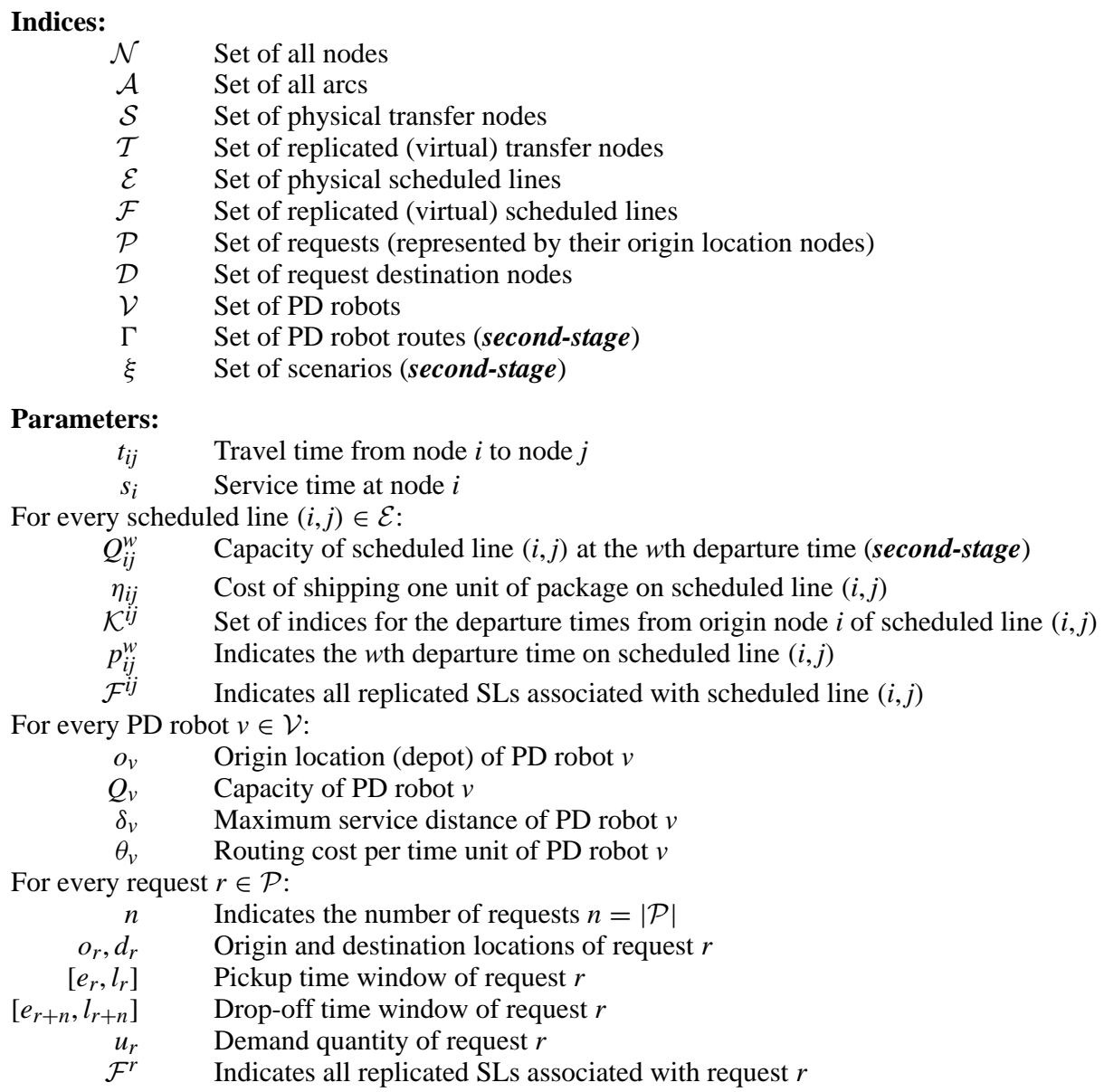

Decision variables:

$$
x_{i j}^{v}=\left\{\begin{array}{ll}
1 & \text { ifarc }(i, j) \text { isusedbyrobot } v \\
0 & \text { otherwise }
\end{array} q_{i j}^{v w}= \begin{cases}1 & \text { ifreplicatedSL }(i, j) \text { isusedbyrobot } v \text { departingfromnode } i \text { attime } p_{i j}^{w} \\
0 & \text { otherwise }\end{cases}\right.
$$

Timing decisions:

$\beta_{i} \quad$ Departure time of a robot from node $i$

$\gamma_{i}^{v} \quad$ Departure time of robot $v \in \mathcal{V}$ from transfer node $i$

Scheduling constraints

$$
\begin{aligned}
& \sum_{v \in \mathcal{V}} x_{i j}^{v}=1 \Longrightarrow \beta_{j} \geq \beta_{i}+t_{i j}+s_{j} \quad \forall i, j \in \mathcal{N} \\
& \beta_{r+n} \geq \beta_{r}+t_{r, r+n}+s_{r+n} \quad \forall r \in \mathcal{P} \\
& e_{i} \leq \beta_{i}-s_{i} \leq l_{i} \quad \forall i \in \mathcal{P} \cup \mathcal{D}
\end{aligned}
$$

Synchronization constraints

$$
\begin{aligned}
& \sum_{w \in \mathcal{K}^{\psi^{i}, \psi^{j}}} q_{i j}^{v w}=x_{i j}^{v} \quad \forall v \in V,(i, j) \in \mathcal{F}^{v} \\
& q_{i j}^{v w}=1 \text { and } x_{i j}^{v}=1 \Longrightarrow \gamma_{i}^{v}=p_{i j}^{w} \quad \forall v \in \mathcal{V},(i, j) \in \mathcal{F}^{v}, w \in \mathcal{K}^{\psi^{i}, \psi^{j}}
\end{aligned}
$$


Decision variable domains

$$
\begin{aligned}
& x_{i j}^{v} \in\{0,1\} \quad \forall(i, j) \in \mathcal{A}, v \in \mathcal{V} \\
& q_{i j}^{v w} \in\{0,1\} \quad \forall v \in \mathcal{V}, \forall(i, j) \in \mathcal{F}^{v}, w \in \mathcal{K}^{\psi^{i}, \psi^{j}} \\
& \beta_{i} \in \mathcal{R}^{+} \quad \forall i \in \mathcal{N} \\
& \gamma_{i}^{v} \in \mathcal{R}^{+} \quad \forall v \in \mathcal{V}, i \in \mathcal{T}
\end{aligned}
$$

The objective function (1) minimises the total costs of operating PD robots and the recourse costs incurred by SL capacity violations. In the recourse function, $\Gamma$ is the given routing vector, $\xi$ is the set of scenarios and $\eta$ is the cost vector for using the scheduled lines per unit shipped. In this problem, we have four sets of constraints: routing, capacity, scheduling and synchronization constraints. As for routing and flow constraints, constraints (2) state that all request pickup and delivery nodes (origins and destinations) are visited exactly once by a PD robot. Constraints (3) ensure that each PD robot must leave its depot at most once. Constraints (4) ensure that each replicated transfer node is visited at most once. Flow conservation for PD robots is considered in constraints (5). Constraints (6) ensure that the same PD robot that picked up the request at its origin will proceed to deliver it to its final destination (i.e. this set of constraints couple the pickup and delivery trips of PD robots). Constraints (7) ensure that the maximum travel distance that PD robots can perform is respected. Since requests demand is known beforehand, constraints (8) ensure that the capacity of PD robots is respected at each time they pickup a request. For the scheduling constraints, constraints (9) ensure that if arc $(i, j)$ is used by PD robot $v$, the departure time of $v$ from node $j$ should be greater than or equal to the sum of $v$ departure time from node $i$, the travel time from $i$ to $j$, and the service time at node $j$. Precedence relations for each request (i.e. request origins should be visited before their destinations) are considered in constraints (10). Constraints (11) enforce time window restrictions on request pickup and delivery. In order to synchronise PD robot trips and the scheduled line, constraints (12) and (13) ensure that the departure time of a PD robot at a transfer node is equal to the SL departure time at that transfer node (i.e. their departures are synchronised).

Note that constraints (9) and (13) are formulated as implications, and thus, need to be linearised. Using standard linearization techniques, we express them by one or two linear inequalities as follows:

$$
\begin{aligned}
& \beta_{j} \geq \beta_{i}+t_{i j}+s_{j}-M\left(1-\sum_{v \in \mathcal{V}} x_{i j}^{v}\right) \quad \forall i, j \in \mathcal{N} \\
& \gamma_{i}^{v} \leq p_{i j}^{w}+M\left(2-q_{i j}^{v w}-x_{i j}^{v}\right) \quad \forall v \in \mathcal{V},(i, j) \in \mathcal{F}^{v}, w \in \mathcal{K}^{\psi^{i}, \psi^{j}} \\
& \gamma_{i}^{v} \geq p_{i j}^{w}-M\left(2-q_{i j}^{v w}-x_{i j}^{v}\right) \quad \forall v \in \mathcal{V},(i, j) \in \mathcal{F}^{v}, w \in \mathcal{K}^{\psi^{i}, \psi^{j}}
\end{aligned}
$$

\subsection{The second-stage decisions}

Due to the uncertainty, the SL capacity might be violated each time a shuttle arrives at a transfer node. This is because passenger demands are unknown by the time of the planning and are assumed to follow a known probability distribution. In other words, the SL service might not be sufficient for the actual passengers demand and PD robots (21). Given the routing solution vector $\Gamma$, indicating PD robot routes and schedules from the first-stage, the aim of the second-stage is to evaluate this solution over a set of scenarios and calculate the expected recourse cost $(E[Q(\Gamma, \xi, \eta)])$. At this stage, a scenario indicates the realised passengers demand at each departure from a transfer node, and thus, the number of available places for transporting PD robots.

$$
\sum_{r \in \mathcal{P}^{\prime}} \sum_{(a, b) \in \mathcal{F}^{i j}} q_{a b}^{r w} \leq Q_{i j}^{w} \quad \forall(i, j) \in \mathcal{E}, w \in \mathcal{K}^{i j}
$$

Since the number of available places at each shuttle is only revealed upon the shuttle's arrival time at a transfer node, capacity violations might occur at the corresponding transfer node (denoted as failure point). Depending on passenger demand realizations, these capacity violations might occur in two different situations:

- Situation\#1: After picking up a request and bringing it to a transfer node, a PD robot may not be able to take the next SL departure at that transfer node due to the high passenger demand (passengers are prioritised over PD robots).

- Situation\#2: After taking a shuttle to travel between two transfer nodes as part of its trip, a PD robot may need to get off the SL at an intermediate transfer node due to high passengers demand. In this case, the PD robot needs to give its place to one of the passengers who are willing to take the SL at that transfer node. 
In both situations, the same capacity violation is obtained: not enough capacity for transporting PD robots with passengers through the SL service. A set of corrective (or recourse) actions needs to be applied in order to recover feasibility, which might lead to additional costs. We consider the following recourse actions to deal with both situations leading to capacity violation outcome. These are:

- Action\#1: If the PD robot cannot take the current departure at the failure point due to high passengers demand, it is transported using the subsequent service of the scheduled line. In other words, the PD robot waits for the next shuttle arriving to the failure point. This recourse action comes with no extra costs as long as waiting the next departure does not violate request delivery time window.

- Action\#2: If waiting for the next shuttle departure leads to violating the capacity of the subsequent SL service or exceeding request delivery time window. If the distance between failure point and request destination is less than the maximum service distance that the PD robot can handle, the PD robot delivers the request to its final destination by itself. This recourse action implies some additional costs since a PD robot might have to perform a longer trip than planned.

- Action\#3: If none of the first two recourse actions can be applied, the request is served by an outsourced service (a dedicated vehicle). This service transports the request from failure point to its final destination. The extra cost implied by using this outsourced service depends on the distance that the outsourced vehicle has to travel.

Another important issue is to rank or schedule PD robots that are waiting to take the scheduled line at one transfer node, according to some criteria. The model needs to decide which PD robots have the priority to be transported in case the realised number of available places in a shuttle is insufficient (situation\#1). For this purpose, we sort PD robots at each transfer node according to the earliest delivery date of the requests they carry. The PD robot carrying request with the earliest delivery date is thus the first to be transported when a shuttle with available places arrives to the corresponding transfer node. A similar issue appears when some PD robots need to get off a shuttle at an intermediate transfer node to give space to more passengers (situation\#2). Therefore, the model also needs to determine the order in which PD robots are asked to get off a shuttle at a certain intermediate transfer node. In this latter case, PD robots already in shuttle are sorted according to their latest delivery date (i.e. PD robot carrying request with the latest delivery date has to get off the shuttle first). It is important to mention that we do not consider the case where a PD robot is asked to get off to allow another one (with an earlier delivery date) to take its place. PD robots are thus asked to get off only to give place to passengers.

To summarise, the recourse function checks if there are capacity violations at each SL departure. In case SL capacity is violated at a given departure, PD robots that are waiting at the corresponding transfer node, referred to as at-node PD robots, are sorted according to the earliest delivery date of their carried requests. In addition, PD robots that are already in-shuttle are sorted according to the latest delivery date of their requests. Then, at-node and in-shuttle PD robots that cannot take the shuttle at the current departure are assigned to the next departure (action\#1) since it does not imply extra costs. If waiting for the next departure leads to violating the request's time windows, the recourse function checks if some PD robots can deliver their requests from the corresponding transfer node to their final destination (action\#2).

Finally, the still remaining requests (i.e. that could not be served using neither action\#1 nor action\#2) are outsourced using dedicated delivery vehicles (action\#3). As a result, depending on actual passenger demand, (i) some at-node PD robots might be able to take SL next departure while others might have to wait, (ii) all at-node PD robots might not be able to take the next shuttle while no in-shuttle PD robots are asked to drop off or (iii) all in-shuttle PD robots may have to drop off from the shuttle and join the waiting PD robots at the corresponding transfer node. The algorithm for calculating the recourse cost of a given routing solution is outlined in Figure 4 (see also Appendix 1 for the detailed recourse function).

\section{Solution approach}

In this section, we present our solution approach. This consists of a scenario-based Sample Average Approximation (SAA) framework (Section 4.1), and an ALNS-based heuristic, to solve the corresponding SAA problems (see Section 4.2).

\subsection{The sample average approximation method}

The SAA method is an iterative approach for solving stochastic optimization problems. It aims at approximating the expected objective function of the stochastic problem using a sample average estimate derived from a random sample (Verweij et al. 2003). While the set of possible scenarios might be very large, the SAA iteratively solves the problem using smaller and more tractable sets of scenarios (referred to as SAA problems), and obtains candidate solutions along with their respective optimality gaps. 


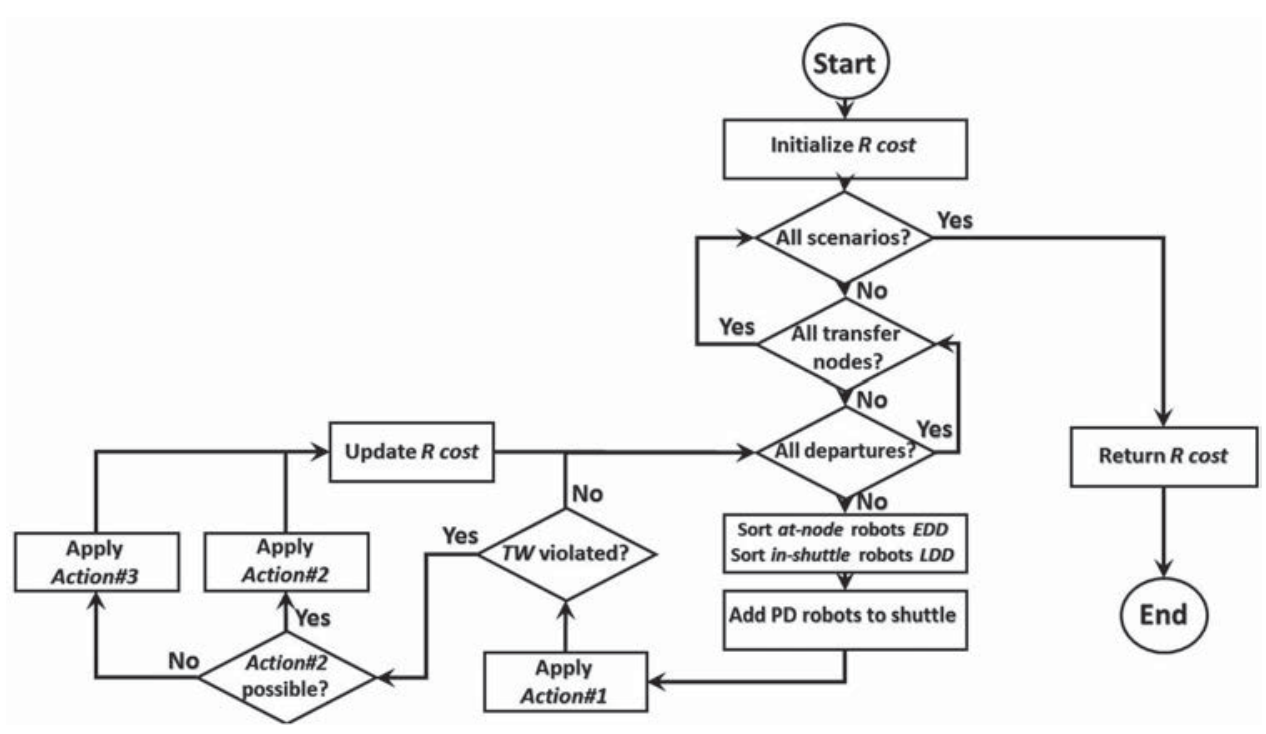

Figure 4. Calculate-recourse-cost algorithm.

The method starts by generating a large set of scenarios $\Omega$ and iterates until the value of the optimal solution is approximated by solving the stochastic problem with smaller sample sets. At each iteration $l$, a sample set of scenarios $\omega_{l}:\left|\omega_{l}\right| \ll|\Omega|$ is generated from the larger set $\Omega$ and the corresponding SAA problem is solved using the ALNS heuristic. The obtained solution $x_{l}$ with objective value $f_{\omega_{l}}^{l}$ is then evaluated using the recourse function (Algorithm 2) in order to determine an upper bound $f_{\Omega}\left(x_{l}\right)$ for the generated set of scenarios $\Omega$ :

$$
f_{\Omega}\left(x_{l}\right)=\text { CalculateRecourseCost }\left(x_{l}, \Omega\right)
$$

Afterwards, a statistical lower bound, denoted by $f_{\omega_{l}}^{\prime}\left(x_{l}\right)$, for the optimal solution value of sample $\omega_{l}$ is calculated by averaging the objective function values obtained in previous iterations:

$$
f_{\omega_{l}}^{\prime}\left(x_{l}\right)=1 / l \sum_{i=1}^{l} f_{\omega_{l}}^{i},
$$

where $f_{\omega_{l}}^{i}$ is the objective function value obtained at iteration $i$. To the best of our knowledge, this is the most commonly used approach in the literature for approximating a statistical lower bound in SAA-based methods (see also Verweij et al. 2003; Ghilas, Demir, and Van Woensel 2016b). Once both bounds are obtained ((22), (23)), the SAA gap is calculated as follows:

$$
\epsilon\left(\omega_{l}, \Omega\right)=f_{\Omega}^{\prime}\left(x_{l}\right)-f_{\omega_{l}}^{\prime}\left(x_{l}\right)
$$

The process continues until the best gap $\epsilon\left(\omega_{l}, \Omega\right)$ is found and the corresponding best solution is returned (see Figure 5, and Appendix B for the detailed algorithm).

\subsection{ALNS heuristic}

An ALNS heuristic algorithm is used to generate routing solutions of minimum total cost. The heuristic is used in combination with the recourse function (Algorithm 2) in order to compute the recourse cost of a generated solution. The main idea of the ALNS is to iteratively apply a set of removal and insertion operators on an initial solution until the best solution is found (Algorithm 1).

The algorithm starts by generating an initial solution indicating initial PD robot routes (see Section 4.2.1). The algorithm then applies a removal operator to remove one PD route from the initial solution. The removed PD route is then reconstructed and reinserted to the solution using an insertion operator and a new solution is obtained. The operators are dynamically selected according to their past and current performances through a roulette-wheel mechanism. In other words, each operator (removal or insertion) is associated with a score that is increased at each time this operator leads to a better solution. These scores reflect how effective operators are in the search progress. Each operator is also associated with a probability that 


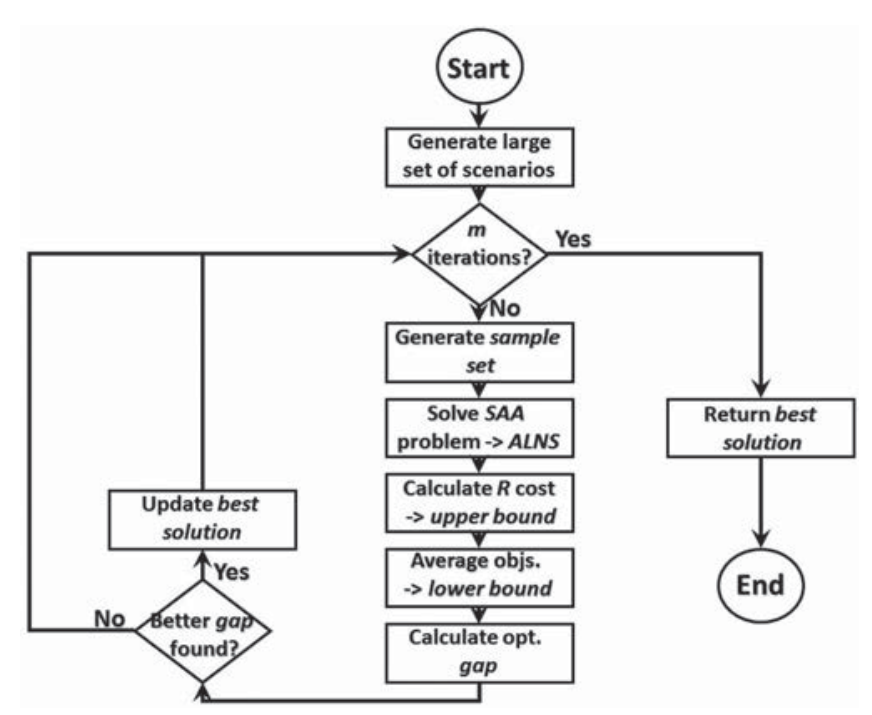

Figure 5. SAA algorithm.

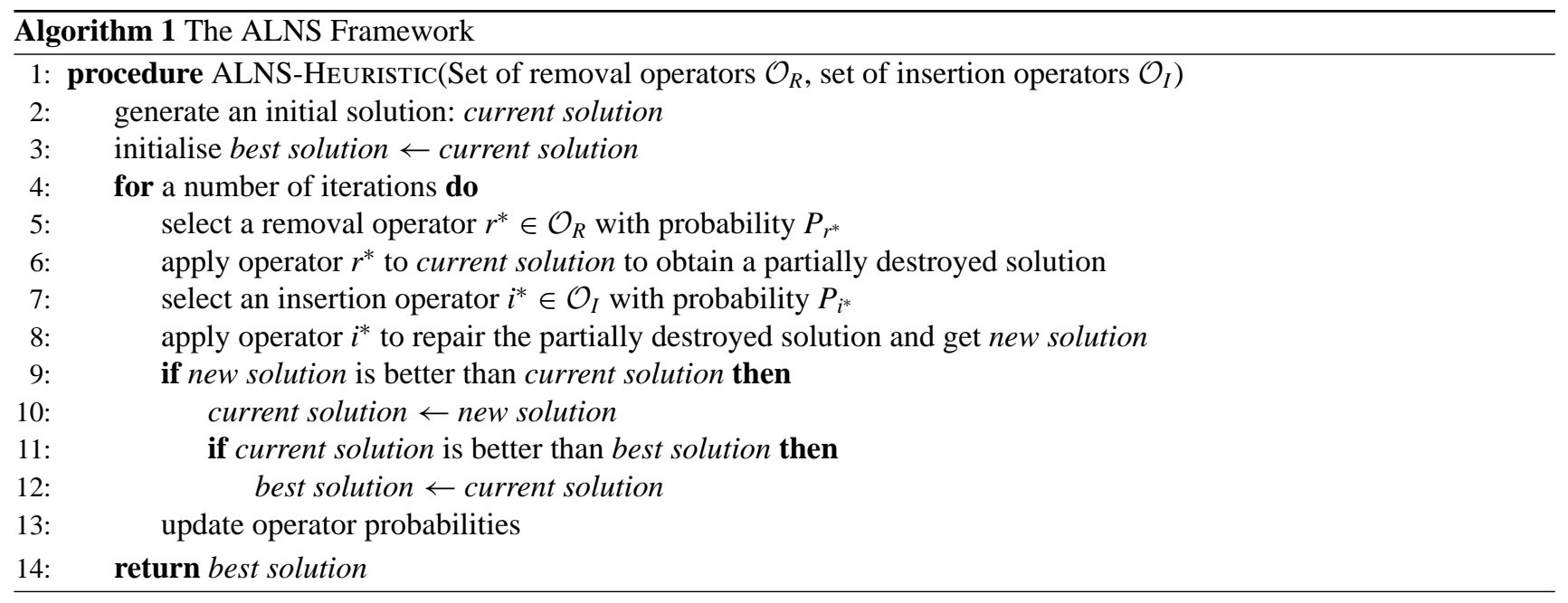

indicates how likely this operator is to be selected (i.e. as the score of an operator increases, its probability of being used in the next iteration becomes higher). This means that operators with better scores have a higher probability to be used by the algorithm. In order to build their scores, operators are selected randomly in the first 100 iterations. The roulette-wheel mechanism is then used based on the calculated operator scores. Once applying these operators yields an improvement, the new solution is stored, and the best solution is updated. The algorithm continues until either a maximum number of iterations or a certain number of iterations with no improvement is reached.

\subsubsection{Generating initial solutions}

Since we use simplified problem settings, in which only one pickup/delivery per PD robot trip is allowed, we start with a simple heuristic to generate initial feasible solutions. This heuristic is composed of two main steps:

(1) We start by selecting requests that can be delivered directly by a PD robot depending on the distance between their origin and destination locations (direct delivery). These direct PD robot routes are then added to the initial solution.

(2) For the other requests (indirect delivery), indirect PD robots are constructed by randomly assigning them to one of the feasible pickup/drop-off transfer nodes while respecting their time restrictions. 
To this end, the feasibility of the returned solution, in terms of request time windows and SL departure times, is assured. This initial feasible solution can then be improved by the ALNS operators as it does not lead to min-cost PD robot routes. ${ }^{3}$ We describe the removal and insertion operators used by the ALNS algorithm in the following sections.

\subsubsection{Removal operators}

- Random removal (R1): This operator removes a randomly selected robot route (request) from the solution which helps in diversifying the search for a better solution.

- Limited random removal (R2): This operator is similar to $R 1$ but it limits the number of times a robot route (request) is removed in the last 100 iterations. For other requests, which their counts have not reached the specified limit, $R I$ is applied.

- Tabu-based removal (R3): This operator also keeps a record of robot route removal counts for the last 100 iterations (as $R 2$ ) and removes those with the smallest frequency of removal rate. This operator also helps in diversifying the search.

- Early-SL-depart removal (R4): This operator removes the robot route with the shortest waiting time at the pickup transfer nodes from the solution. The request waiting time at a specific transfer node is obtained from the difference between its arrival to that transfer node and its departure from it (i.e. a PD robot might have to wait at a transfer node until the next SL departure).

- Late-SL-depart removal (R5): Unlike $R 4$, this operator removes the robot route with the longest waiting time at transfer nodes from the solution.

\subsubsection{Insertion operators}

- Pickup Transfer-node insertion (I1): This operator reconstructs a robot route by assigning it to a different pickup transfer node than the one it was assigned to before being removed. This operator helps diversifying the search by leading to different transportation costs (i.e. operational and recourse costs). This potential improvement highly depends on SL and PD robot transshipment costs as well as the maximum service distance of PD robots which can limit the feasibility of this assignment.

- Drop-off Transfer-node insertion (I2): This operator reconstructs a robot route by assigning it to a drop-off transfer node that is different than the one it was assigned to before being removed. Similar to $I 1$, this operator can lead to different transportation costs.

- Early-SL-depart insertion (I3): This operator reconstructs a robot route by assigning it to the same pickup transfer node but with an earlier departure time. Indeed, changing the SL departure to which a PD robot is assigned leads to different recourse costs as passengers demand varies between different SL departures.

- Late-SL-depart insertion (I4): Unlike I3, this operator reconstructs a robot route by assigning it to the same pickup transfer node but with a later departure time. This operator is also important for solving the stochastic optimization problem as it leads to different recourse costs.

That said, these operators are used by the heuristic to remove and insert robot routes to a current solution. They provide a reasonable choice for our problem settings where only one request is served during a PD robot trip. The heuristic can thus be extended by considering different operators when PD robots are allowed to perform multiple pickups and deliveries at one trip (see operators at Ghilas, Demir, and Van Woensel 2016a, 2016b).

\section{Computational study}

In this section, an extensive computational study to assess the performance of the proposed solution approach is presented. First, we explain how we generate test instances and we describe different parameters used (see Section 5.1). We then show how we generate the set of scenarios used by the SAA algorithm for solving the stochastic problem (see Section 5.2). Afterwards, we analyse the performance of the proposed heuristic approach along with the different operators used and compare the results obtained from solving the stochastic problem with those of the deterministic one (i.e. when no uncertainty is considered). Finally, we study the impacts of the considered source of uncertainty on the obtained solutions with different settings (see Section 5.3).

\subsection{Parameters and instance generation}

For testing the proposed solution approach, we generate instances with different network topologies and freight request distributions. Generated instances are named as P_D_r_n, where P represents the network topology, D is the geographical 


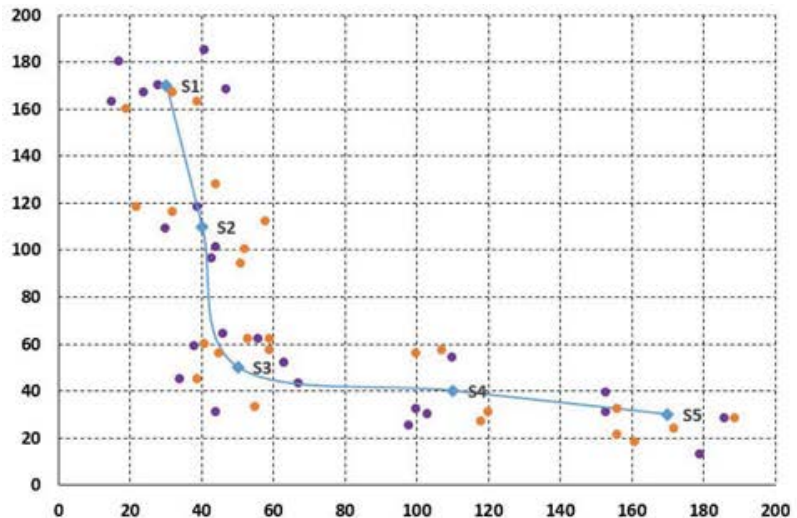

(a)

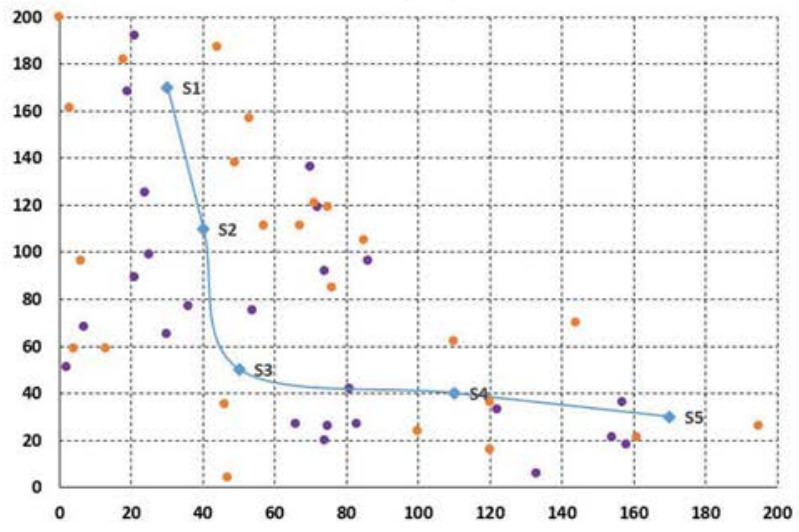

(c)

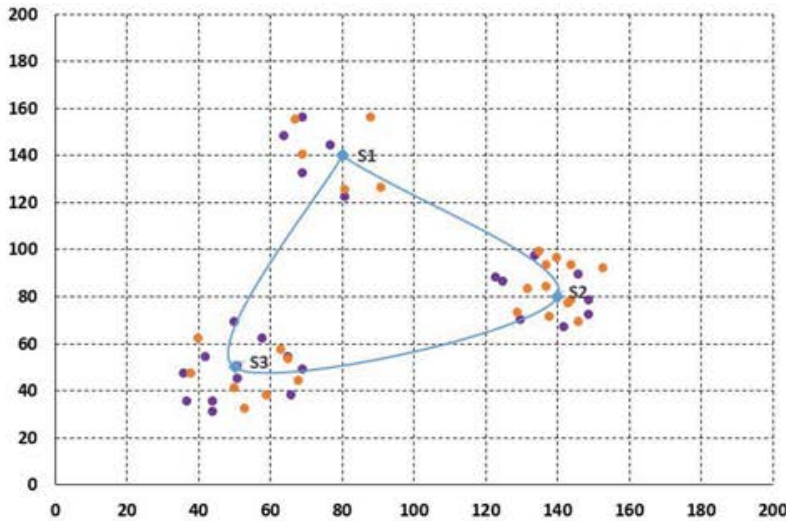

(b)

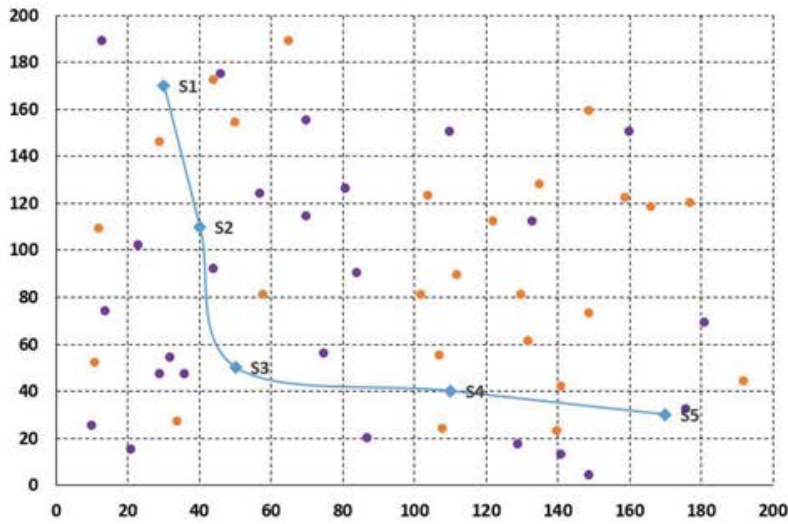

(d)

Figure 6. Instances with different network topologies and request distributions: (a) L_C_30_25, (b) T_C_30_25, (c) L_RC_50_25, (d) L_UR_50_25.

distribution of freight requests, $r$ is request nodes range from transfer nodes and $n$ is the number of freight requests. Since the proposed model can adapt different network topologies, we generate instances with line (referred to as ' $\mathrm{L}$ ') and triangular (referred to as ' $T$ ') topologies (Figure 6a,b). While the number of SLs is different, instances with either topology have the same characteristics. In addition, each instance contains up to 60 freight requests where their origin and destination nodes are distributed over $200 \times 200$ Euclidean space. We consider three different distributions of freight requests (inspired from Ghilas, Demir, and Van Woensel 2016b). These are: C - freight request origin and destination nodes are clustered within at most 30 time units around transfer nodes (Figure 6a), $\mathrm{RC}$ - request nodes are randomly clustered within at most 50 time units to one of the available transfer nodes (Figure 6c), and UR - freight requests are uniform-randomly distributed over the considered space (Figure 6d). As PD robots are located at transfer nodes, we consider up to three PD robots at each transfer node.

We consider a planning horizon of 600 time units where SL departure interval is set to 30 time units (i.e. there is a shuttle departing from each transfer node every 30 time units). We consider that this frequency is enough to cover passengers demand through SL. We generate request pickup and delivery time windows randomly with an average width of 40 time units. A minimum of 100 time units is also assured between the end of pickup time window and the start of delivery time window. Service time at each location (i.e. pickup, drop-off or transfer node locations) is set to three time units. This service time represents the time needed for a PD robot to pick up or deliver a freight request, or to get in or off a shuttle at a transfer node.

The capacity of each PD robot and the quantity of each freight request are set to 1 . This means that each PD robot can serve one freight (i.e. any freight request) at a time. The capacity of shuttles on SL is set to 10 places for both passengers and PD robots where PD robots can take up to 3 places (different limits are analysed in Section 5.3). Regarding transportation costs, we assume the time unit cost for PD robots to be 0.5 unit. This cost includes energy consumption, insurance and 
Table 2. Set of parameters used in the computational study.

\begin{tabular}{lclc}
\hline Parameter & Value & \multicolumn{1}{c}{ Parameter } & Value \\
\hline PD robot cost & 0.5 & Num. iterations no improvement & 50 \\
SL cost & 1 & The size of the large set of scenarios & 10,000 \\
Outsourcing cost & 3 & The size of the sample set of scenarios & 50 \\
PD robot capacity & 1 & Number of ALNS iterations & 10,000 \\
SL capacity & 10 & Number of SAA iterations & 10 \\
Max. num. places for PD robots & 3 & Score for new best solution & 3 \\
Freight request quantity & 1 & Score for improving the current solution & 1 \\
\hline
\end{tabular}

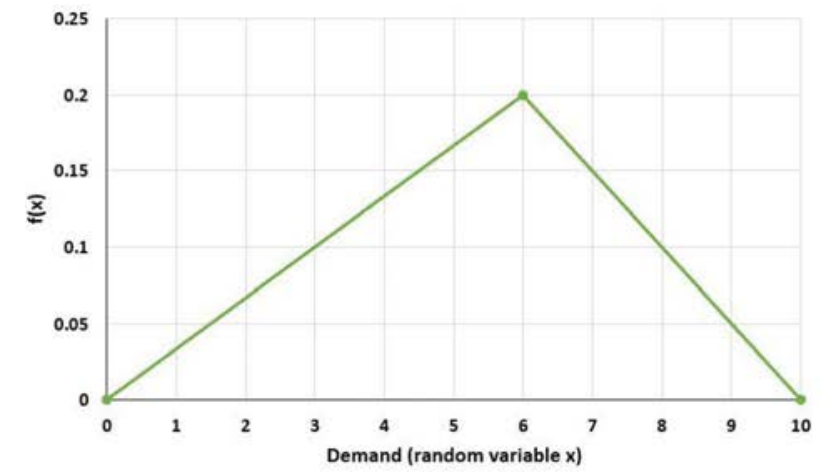

(a)

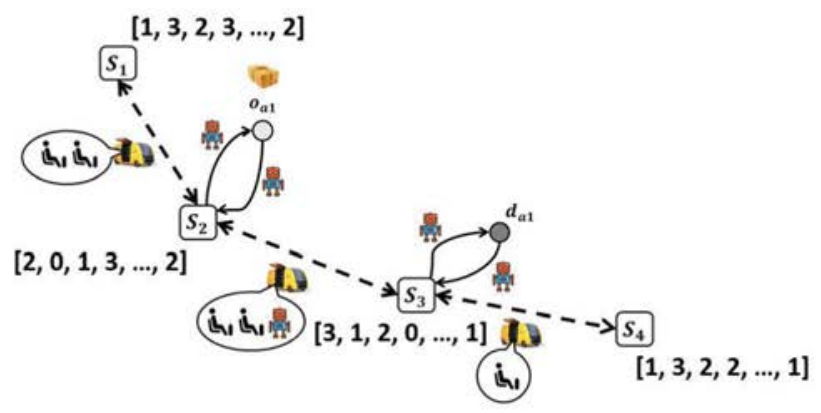

(b)

Figure 7. SAA scenario generation: (a) passengers demand distribution and (b) an example of SAA scenario.

transportation expenses induced when PD robots are used. In addition, the time unit cost of using SL service is set to 1 unit. This cost includes loading, unloading and transportation expenses each time a PD robot uses the SL service. Finally, the recourse cost of using the outsource delivery service is assumed to be three units (different SL and robot shipment costs were analysed in Ghilas, Cordeau, and Demir 2016).

As introduced in Section 4.2, we consider two stopping criteria for the heuristic method. These are: the maximum number of ALNS iterations which is set to 10,000 iterations and the maximum number of consecutive iterations with no improvement which is set to 50 iterations. In addition, the score of an operator is increased by 1 if it leads to improving the current solution, and by 3 if a new best solution is found. For the SAA algorithm, the size of the large set of scenarios $(\Omega)$ is set to 10000 scenarios while the size of the smaller sample $(\omega)$ is set to 50 scenarios. Finally, the number of SAA iterations is set to 10 (SAA parameters are fixed based on Li et al. 2016b; Ghilas, Demir, and Van Woensel 2016b where similar problems and solution methods are considered). The set of parameters used in the computational study along with their values are presented in Table 2.

\subsection{Scenario generation}

In order to test the proposed SAA algorithm, we need to generate a large set of scenarios which represent the realised passengers demand at each SL departure. The actual passengers demand helps the algorithm to decide whether PD robots can be transported through SLs or some recourse actions need to be applied. For this purpose, passengers demand is assumed to follow a discrete triangular distribution for a given minimum value $a=0$, mean $b=6$ and a maximum value $c=10$ (Figure 7a).

For example in Figure 7(b), based on the realised passengers demand, the number of available places for PD robots at the different departures of scheduled line $S_{2} \rightarrow S_{3}$ is respectively $[2,0,1,3, \ldots, 2]$. Hence, PD robots are not able to take the second shuttle departure from $S_{2}$ to $S_{3}$ as there are no available places for them, while there are three available places at the fourth departure, etc.

\subsection{Experiments}

The algorithms developed in this paper (i.e. recourse, ALNS and SAA algorithms) are implemented in Java 1.8.0. CPLEX 12.6 solver is used for solving the MIP formulation. Instances are tested on a quad-core i5-5300U machine with 8 GB of 
Table 3. Analysing ALNS performance.

\begin{tabular}{|c|c|c|c|c|c|c|c|}
\hline Instance & \# dir. & \# ind. & \# usv. & Cost & Gap (\%) & CPU (s) & \# iter. \\
\hline L_C_30_10 & $0(\mathbf{0})$ & $10(\mathbf{1 0})$ & $0(\mathbf{0})$ & 1030.1 (1030.1) & 0.0 & 0.03 (4.1) & 19 \\
\hline L_C_30_20 & $2(2)$ & 18 (18) & $0(\mathbf{0})$ & 3339.1 (3339.1) & 0.0 & $0.31(\mathbf{3 2 . 3})$ & 269 \\
\hline L C C $30 \quad 30$ & $3(3)$ & 27 (27) & $0(\mathbf{0})$ & 4156.4 (4156.4) & 0.0 & 0.53 (143.5) & 431 \\
\hline L_C_30_40 & $5(5)$ & $35(35)$ & $0(\mathbf{0})$ & 4896.9 (4872.2) & 0.49 & 0.79 (537.6) & 728 \\
\hline L_C_30_60 & $7(7)$ & $53(53)$ & $0(\mathbf{0})$ & 7452.2 (7443.9) & 0.11 & 1.47 (3103.4) & 949 \\
\hline L_C_30_100 & $12(-)$ & $88(-)$ & $0(-)$ & $12695.1(-)$ & - & $5.01(-)$ & 3157 \\
\hline L_RC_30_10 & $2(2)$ & $8(8)$ & $0(\mathbf{0})$ & 971.2 (971.2) & 0.0 & $0.03(\mathbf{3 . 3})$ & 20 \\
\hline L_RC_30_20 & $2(2)$ & 18 (18) & $0(\mathbf{0})$ & 2910.4 (2910.4) & 0.0 & 0.26 (23.9) & 219 \\
\hline L_RC_30_30 & $3(3)$ & $25(\mathbf{2 5})$ & $2(2)$ & $4120.2(\mathbf{4 1 2 0 . 2})$ & 0.0 & 0.41 (112.9) & 382 \\
\hline L_RC_30_40 & $5(5)$ & 34 (34) & $1(\mathbf{1})$ & $5291.3(\mathbf{5 2 9 1 . 3})$ & 0.0 & 0.76 (351.9) & 656 \\
\hline L_RC_30_60 & $8(8)$ & $51(51)$ & $1(\mathbf{1})$ & 9006.8 (8983.8) & 0.25 & 1.53 (1802.4) & 976 \\
\hline L_RC_30_100 & $12(-)$ & $87(-)$ & $1(-)$ & $14747.9(-)$ & - & $5.31(-)$ & 3323 \\
\hline L_UR_30_10 & $0(\mathbf{0})$ & $8(8)$ & $2(2)$ & 1472.1 (1472.1) & 0.0 & $0.03(\mathbf{4 . 5})$ & 17 \\
\hline L_UR_30_20 & $1(1)$ & $16(\mathbf{1 6})$ & $3(3)$ & $3510.0(\mathbf{3 5 1 0 . 0})$ & 0.0 & 0.32 (26.9) & 228 \\
\hline L_UR_30_30 & $2(2)$ & $23(\mathbf{2 3})$ & $5(5)$ & 4707.5 (4707.5) & 0.0 & 0.49 (135.7) & 431 \\
\hline L_UR_30_40 & $1(1)$ & 34 (34) & $5(5)$ & 6120.8 (6099.7) & 0.34 & 0.69 (349.3) & 547 \\
\hline L_UR_30_60 & $4(4)$ & $51(51)$ & $5(5)$ & 9779.1 (9741.8) & 0.38 & 1.57 (2215.9) & 1095 \\
\hline L_UR_30_100 & $5(-)$ & $85(-)$ & $10(-)$ & $14917.4(-)$ & - & $6.12(-)$ & 4217 \\
\hline T_C_30_10 & $0(\mathbf{0})$ & $10(\mathbf{1 0})$ & $0(\mathbf{0})$ & 1087.7 (1087.7) & 0.0 & $0.03(\mathbf{1 . 3})$ & 21 \\
\hline T_C_30_20 & $5(5)$ & $15(\mathbf{1 5})$ & $0(\mathbf{0})$ & 1826.9 (1826.9) & 0.0 & $0.14(7.1)$ & 239 \\
\hline T_C_30_30 & $7(7)$ & $23(\mathbf{2 3})$ & $0(\mathbf{0})$ & 2535.9 (2535.9) & 0.0 & 0.47 (31.1) & 341 \\
\hline T_C_30_40 & $7(7)$ & $33(33)$ & $0(\mathbf{0})$ & 4087.4 (4063.5) & 0.0 & 1.07 (95.4) & 672 \\
\hline T_C_30_60 & 12 (12) & $48(48)$ & $0(\mathbf{0})$ & 6001.2 (5978.6) & 0.38 & 1.62 (444.9) & 1313 \\
\hline T_C_30_100 & $20(-)$ & $79(-)$ & $1(-)$ & $9473.3(-)$ & - & $4.37(-)$ & 3543 \\
\hline T_RC_30_10 & $2(2)$ & $7(7)$ & $1(\mathbf{1})$ & 1121.3 (1121.3) & 0.0 & $0.03(\mathbf{1 . 2})$ & 19 \\
\hline T_RC_30_20 & $1(1)$ & 17 (17) & $2(2)$ & 2224.4 (2224.4) & 0.0 & $0.22(\mathbf{5 . 9})$ & 227 \\
\hline T_RC_30_30 & $3(3)$ & $24(24)$ & $3(3)$ & 3666.8 (3666.8) & 0.0 & $0.39(\mathbf{2 4 . 5})$ & 318 \\
\hline T_RC_30_40 & $1(\mathbf{1})$ & $36(36)$ & $3(3)$ & 5126.3 (5126.3) & 0.0 & $0.96(61.7)$ & 566 \\
\hline T_RC_30_60 & $6(6)$ & $51(\mathbf{5 1})$ & $3(3)$ & 6340.7 (6303.1) & 0.58 & 1.61 (371.7) & 1032 \\
\hline T_RC_30_100 & $11(-)$ & $84(-)$ & $5(-)$ & $10148.9(-)$ & - & $5.91(-)$ & 3782 \\
\hline T_UR_30_10 & $0(\mathbf{0})$ & $7(7)$ & $3(3)$ & 1164.6 (1164.6) & 0.0 & 0.03 (1.2) & 24 \\
\hline T_UR_30_20 & $1(1)$ & $16(16)$ & $3(3)$ & 2784.6 (2784.6) & 0.0 & $0.19(\mathbf{5 . 8})$ & 188 \\
\hline T_UR_30_30 & $2(2)$ & $24(24)$ & $4(4)$ & 3825.7 (3825.7) & 0.0 & $0.32(\mathbf{2 0 . 6})$ & 240 \\
\hline T_UR_30_40 & $2(2)$ & $34(34)$ & $4(4)$ & 5673.4 (5642.6) & 0.54 & 0.94 (61.6) & 643 \\
\hline T_UR_30_60 & $5(5)$ & $44(44)$ & $11(\mathbf{1 1})$ & 7861.9 (7829.2) & 0.41 & 1.38 (317.6) & 927 \\
\hline T_UR_30_100 & $8(-)$ & $78(-)$ & $14(-)$ & $13351.1(-)$ & - & $4.34(-)$ & 2976 \\
\hline Average ALNS & 4.6 & 36.1 & 2.5 & 5539.6 & 0.116 & 1.38 & 937.6 \\
\hline
\end{tabular}

RAM. We study the efficiency of the proposed ALNS approach by comparing its results to those obtained by solving the first-stage model (introduced in Section 3.1) with CPLEX solver and analysing the performance of its operators. We then examine the stochastic solutions obtained by the SAA algorithm, compare them with the deterministic ones, and analyse the impact of different levels of passengers demand, SL frequency and capacity on the obtained solutions.

\subsubsection{Analysing ALNS performance}

The results of solving instances with up to 100 freight requests are presented in Table 3 (results obtained by CPLEX are in bold). In this table, \# dir. indicates the number of direct deliveries, \# ind. indicates the number of indirect deliveries and \# usv. indicates the number of unserved freight requests. In addition, Cost column represents the total transportation costs obtained by the ALNS heuristic and CPLEX (respectively) while Gap (\%) column gives the optimality gap percentage between them. Finally, CPU column indicates the execution time needed to run both approaches and \# iter. column gives the number of ALNS iterations performed.

Looking at Table 3, we observe that the proposed ALNS is always able to find a solution that is identical to the optimal one obtained by solving the MIP in terms of direct and indirect deliveries. In addition, the ALNS is able reach the optimal solutions $(G a p=0)$ for all instances with less than 40 freight requests. For instances with more than 40 requests, the ALNS is still capable of finding solutions that are within $0.6 \%$ of the optimal solutions. Moreover, the proposed heuristic returns solutions for instances with 100 requests for which CPLEX is not able to find optimal solutions. This is due to the increasing complexity of the problem (i.e. number of variables) when the number of freight requests gets larger. Since the numbers of 
Table 4. The performance of removal operators.

\begin{tabular}{lccccc}
\hline Instance & R1 & R2 & R3 & R4 & R5 \\
\hline L_C_30_60 & $25.4 \%(0.02)$ & $23.1 \%(0.02)$ & $23.6 \%(0.02)$ & $14.1 \%(0.01)$ & $13.8 \%(0.01)$ \\
L_RC_30_60 & $24.3 \%(0.02)$ & $27.2 \%(0.02)$ & $21.4 \%(0.02)$ & $12.6 \%(0.01)$ & $14.5 \%(0.01)$ \\
L_UR_30_60 & $22.1 \%(0.02)$ & $24.5 \%(0.02)$ & $24.1 \%(0.02)$ & $16.8 \%(0.01)$ & $12.5 \%(0.01)$ \\
T_C_30_60 & $26.5 \%(0.02)$ & $25.3 \%(0.02)$ & $22.7 \%(0.02)$ & $12.1 \%(0.01)$ & $13.4 \%(0.01)$ \\
T_RC_30_60 & $26.2 \%(0.02)$ & $23.1 \%(0.02)$ & $27.6 \%(0.02)$ & $12.9 \%(0.01)$ & $10.2 \%(0.01)$ \\
T_UR_30_60 & $24.9 \%(0.02)$ & $23.9 \%(0.02)$ & $25.2 \%(0.02)$ & $11.8 \%(0.01)$ & $14.2 \%(0.01)$ \\
Average & $\mathbf{2 4 . 9 \%}$ & $\mathbf{2 4 . 5 \%}$ & $\mathbf{2 4 . 1 \%}$ & $13.4 \%$ & $13.1 \%$ \\
\hline
\end{tabular}

Table 5. The performance insertion operators.

\begin{tabular}{lcccc}
\hline Instance & I1 & I2 & I3 & I4 \\
\hline L_C_30_60 & $34.3 \%(0.04)$ & $37.8 \%(0.04)$ & $15.4 \%(0.02)$ & $12.5 \%(0.01)$ \\
L_RC_30_60 & $32.7 \%(0.04)$ & $38.4 \%(0.04)$ & $14.8 \%(0.01)$ & $14.1 \%(0.01)$ \\
L_UR_30_60 & $28.9 \%(0.03)$ & $36.8 \%(0.04)$ & $16.3 \%(0.02)$ & $16.1 \%(0.02)$ \\
T_C_30_60 & $31.5 \%(0.04)$ & $38.3 \%(0.04)$ & $13.6 \%(0.01)$ & $13.9 \%(0.01)$ \\
T_RC_30_60 & $34.7 \%(0.04)$ & $40.1 \%(0.04)$ & $16.4 \%(0.02)$ & $11.6 \%(0.01)$ \\
T_UR_30_60 & $33.8 \%(0.04)$ & $35.2 \%(0.04)$ & $15.8 \%$ & $14.6 \%(0.01)$ \\
Average & $\mathbf{3 2 . 6 \%}$ & $\mathbf{3 7 . 8 \%}$ & & $13.8 \%$ \\
\hline
\end{tabular}

direct and indirect deliveries are the same in both solutions, this small gap indicates that there are very few requests that could have been assigned to another pickup or drop-off transfer node so that some costs can be saved. We also observe that total costs are generally lower for instances with clustered request distribution (L_C \& T_C). This can be explained by the likelihood of performing direct deliveries which is higher in clustered instances, while requests are more scattered in randomly distributed instances (Table 3, '\# dir.'). This can also be reflected by the increasing number of unserved requests in randomly distributed instances. In this latter case, some requests cannot be brought to transfer nodes due to PD robot distance limitations. Another observation is that the total costs are generally higher in line networks than in triangular ones. This indicates that a triangular network might provide a better coverage to the service area while reducing transportation costs.

The base case instances, with 10 freight requests, solve in few seconds with CPLEX. This amount of time increases as the number of freight requests increases. We observe that instances with line network topology need longer time to be solved to optimality than those with triangular topology (an average of $6.3 \mathrm{~min}$ for triangular instances with 60 requests compared to $39.5 \mathrm{~min}$ for same instances with line topology). The reason is that the number of transfer nodes, and thus the number of variables and graph edges, is bigger in instances with line topologies. This observation also gives an indication that a triangular network topology might be more effective in terms of computational efforts needed to solve its instances. On the other hand, the proposed heuristic solves the different instances in very short running times ( $1.38 \mathrm{~s}$ in average) while maintaining near-optimal solutions. These short running times suggest that our approach is suitable for approximating optimal solutions for the stochastic problem where instances have to be solved over a large set of scenarios in the SAA method.

In Tables 4 and 5, we analyse the removal and insertion operators used in the ALNS using some relevant information on their performance. For each operator, we present its usage frequency as a percentage of the total number of iterations, and the total time spent on running it (given in parenthesis).

Considering removal operators (Table 4), we observe that operators R1, R2 and R3 are the most frequently used. This is mainly because these three operators randomly select robot routes (requests) and are used to diversify the search for a better solution.

We also observe that I1 and I2 are the most frequently used insertion operators (Table 5). This indicates that operators which assign robot route to an earlier, or later, SL departure (i.e. I3 and I4) are used less than other operators which assign it to a different pickup, or drop-off, transfer node.

\subsubsection{Analysing SAA performance and stochastic solutions}

In order to quantify the impact of stochastic passengers demand, we solve the instances, introduced earlier in Table 3, using the proposed SAA algorithm. Results are presented in Table 6 where the first three columns represent the usage frequency 
Table 6. SAA results.

\begin{tabular}{|c|c|c|c|c|c|c|}
\hline Instance & $\operatorname{act} 1(\%)$ & $\operatorname{act} 2(\%)$ & $\operatorname{act} 3(\%)$ & Cost (oper., rcs.) & $\operatorname{Add}(\%)$ & $\mathrm{CPU}(\mathrm{s})$ \\
\hline L_C_30_10 & 95 & 1 & 4 & $\mathbf{1 0 6 6 . 2}(1019.1,47.1)$ & 3.5 & 15.7 \\
\hline L_C_30_20 & 81 & 2 & 17 & $\mathbf{3 4 2 7 . 1}(3070.2,356.9)$ & 2.6 & 154.8 \\
\hline L_C_30_30 & 86 & 1 & 13 & $4350.3(3815.3,534.9)$ & 4.7 & 234.5 \\
\hline L_C_30_40 & 91 & 2 & 8 & $4983.4(4382.8,600.6)$ & 2.3 & 395.2 \\
\hline L_C_30_60 & 89 & 0.5 & 1.5 & $7498.4(7382.5,115.8)$ & 0.7 & 635.4 \\
\hline L_C_30_100 & 94 & 1 & 5 & 12839.7 (12671.6, 168.1) & 1.1 & 2505.2 \\
\hline L_RC_30_10 & 99 & 1 & 0 & $975.1(970.7,4.5)$ & 0.4 & 13.8 \\
\hline L_RC_30_20 & 85 & 0 & 15 & $3257.1(2924.8,332.2)$ & 5.7 & 129.8 \\
\hline L_RC_30_30 & 92 & 0 & 8 & $4224.6(4119.4,105.2)$ & 2.5 & 204.5 \\
\hline L_RC_30_40 & 98 & 0 & 2 & 5326.1 $(5219.6,106.5)$ & 0.7 & 381.2 \\
\hline L_RC_30_60 & 97 & 1 & 2 & $\mathbf{9 2 2 1 . 7}(8901.9,319.8)$ & 2.6 & 765.1 \\
\hline L_RC_30_100 & 95 & 1 & 4 & 15322.6 (14356.7, 965.9) & 3.9 & 2655.4 \\
\hline L_UR_30_10 & 99 & 1 & 0 & $1478.3(1470.1,8.3)$ & 0.4 & 12.5 \\
\hline L_UR_30_20 & 93 & 1 & 6 & $3559.3(3427.8,131.5)$ & 1.4 & 157.4 \\
\hline L_UR_30_30 & 94 & 1 & 5 & $4960.1(4622.6,337.6)$ & 5.3 & 244.1 \\
\hline L_UR_30_40 & 98 & 0 & 2 & $\mathbf{6 1 8 3 . 1}(6165.2,17.8)$ & 1.4 & 345.5 \\
\hline L_UR_30_60 & 98 & 1 & 1 & $9929.2(9670.2,258.9)$ & 1.9 & 758.1 \\
\hline L_UR_30_100 & 96 & 0 & 4 & 15464.1 (14619.4, 844.6) & 3.7 & 3060.3 \\
\hline T_C_30_10 & 97 & 3 & 0 & $1172.9(1170.6,2.3)$ & 7.8 & 14.8 \\
\hline T_C_30_20 & 85 & 1 & 14 & $2058.4(1789.6,268.4)$ & 8.1 & 72.4 \\
\hline T_C_30_30 & 97 & 0 & 3 & $2582.9(2483.6,99.3)$ & 1.9 & 233.1 \\
\hline T_C_30_40 & 94 & 2 & 4 & 4213.2 $(4079.8,133.3)$ & 3.1 & 535.4 \\
\hline T_C_30_60 & 92 & 1 & 7 & $\mathbf{6 1 1 4 . 8}(5669.5,445.2)$ & 2.3 & 810.1 \\
\hline T_C_30_100 & 97 & 1 & 2 & $9589.1(9424.9,164.1)$ & 1.2 & 2185.7 \\
\hline T_RC_30_10 & 91 & 6 & 3 & $1138.7(1074.3,72.4)$ & 1.6 & 12.9 \\
\hline T_RC_30_20 & 96 & 3 & 1 & $2270.6(2208.6,62.4)$ & 2.1 & 112.4 \\
\hline T_RC_30_30 & 94 & 1 & 5 & $3738.8(3578.6,160.2)$ & 1.9 & 196.2 \\
\hline T_RC_30_40 & 90 & 2 & 8 & $\mathbf{5 5 6 6 . 4}(5041.7,524.7)$ & 8.6 & 581.3 \\
\hline T_RC_30_60 & 93 & 1 & 6 & $\mathbf{6 6 7 8 . 8}(6231.9,446.9)$ & 5.9 & 805.7 \\
\hline T_RC_30_100 & 97 & 1 & 2 & $\mathbf{1 0 4 0 7 . 3}(10045.2,362.1)$ & 2.6 & 2955.2 \\
\hline T_UR_30_10 & 95 & 0 & 5 & $1216.1(1151.2,64.9)$ & 4.4 & 12.8 \\
\hline T_UR_30_20 & 97 & 0 & 3 & $2828.3(2763.9,64.4)$ & 1.6 & 96.1 \\
\hline T_UR_30_30 & 91 & 1 & 8 & $4109.7(3736.7,372.9)$ & 7.4 & 162.4 \\
\hline T_UR_30_40 & 97 & 0.5 & 2.5 & $\mathbf{5 7 0 4 . 1}(5626.9,77.1)$ & 1.1 & 570.3 \\
\hline T_UR_30_60 & 94 & 1 & 5 & $8159.8(7732.6,427.1)$ & 4.2 & 691.6 \\
\hline T_UR_30_100 & 95 & 1 & 4 & $13893.2(13207.2,686.1)$ & 3.9 & 2170.8 \\
\hline Average SAA & 93.6 & 1.1 & 5.3 & $\mathbf{5 7 0 8 . 6}(5439.6,269.2)$ & 3.3 & 691.3 \\
\hline
\end{tabular}

of recourse actions 1, 2 and 3 (respectively) as a percentage of the total number of times recourse actions were used for each instance. The total transportation cost is then given along with the associated operational and recourse costs. The additional cost induced by uncertainty is then calculated by comparing the total cost of the stochastic solution with that of solving the deterministic version of the problem using the heuristic (given in Table 3).

In Table 6, we observe that the realization of passengers demand can add an average of $3.3 \%$ to the total transportation cost. This increase is due to the recourse actions that are used to correct the interrupted robot routes. Indeed, when passengers demand is revealed, the actual number of places for PD robots at each SL departure might not be sufficient and recourse actions need to be applied adding extra expenses to the total transportation cost. Since the recourse function applies recourse actions one by one to recover feasibility, one can observe that action\#l is the most frequently used among the other recourse actions (93.6\% in average). This is because this recourse action uses the subsequent SL service (i.e. waiting the next SL departure) which does not imply additional transportation costs. We also observe that action\#2 is not frequently used by the algorithm (only 1.1\%). This indicates that a direct PD robot delivery, from failure point to request destination, is not feasible in most of the time due to PD robot distance limitations. Most of the added recourse costs are thus induced by action\#3 as it guarantees the feasibility of all interrupted deliveries using the outsourced service. Regarding network topology, we observe that the average added cost for instances with line topology is lower than those with triangular topology (2.5\% compared to $3.8 \%$ ). This is because the number of stations in triangular network is less than that of the line network. As a consequence, the number of PD robots at each station is larger in a triangular network and the likelihood of applying recourse actions (i.e. to recover capacity violations at each SL departure) is thus higher. 
Table 7. Different TW width.

\begin{tabular}{lcccccccc}
\hline & \multicolumn{2}{c}{ TW40 } & & \multicolumn{2}{c}{ TW30 } & & \multicolumn{2}{c}{ TW20 } \\
\cline { 2 - 3 } & Cost & CPU(s) & & Cost & CPU(s) & & Cost & CPU(s) \\
\hline Average & 7488.5 & 635.4 & & 7561.5 & 663.7 & & 7654.8 & 698.2 \\
\hline
\end{tabular}

In addition, we compare our system to that considered in Ghilas, Demir, and Van Woensel (2016b) where a similar problem and solution approach were proposed. In Ghilas, Demir, and Van Woensel (2016b), an SAA approach combined with an ALNS is used to solve a standard pickup and delivery problem with scheduled lines (PDPTW-SL) where multiple pickups and deliveries are allowed and demand quantities are stochastic. This setting is reasonable as they use vehicles to perform the pickup and delivery process. Indeed, vehicles can operate for longer distances and can accommodate multiple requests simultaneously compared to PD robots. In our case, only a single pickup/delivery is allowed per robot trip. This is because PD robots have limited distance and storage capabilities (i.e. a PD robot picks up or delivers one freight request at a time and returns to the station). This suggests that generating routes for PD robots requires less sophisticated operators, and thus, less computational effort than building routes for vehicles. In addition, the number of possible scenarios in our case depends on the number of SL departures at each station. In other words, the number of scenarios does not change when the number of requests increases while request quantities are identical. In their study, as demand quantities are stochastic, the number of scenarios highly depends on the size of the considered instance and increases as the number of requests becomes higher. However, we consider an extended recourse function where PD robots (carrying requests) might not be able to use SL service or even have to get-off at some point due to high passengers demand. This increases the difficulty of the considered stochastic problem. Having said this, the problem we consider can be classified as a stochastic variant of the PDPTW-SL problem (introduced in Ghilas, Cordeau, and Demir 2016) with single pickup and delivery. The PDPTW-SL is an extension of the classical PDPTW which is NP-hard in general (Tan and Huang 2019). This is due to time windows which make the problem of finding a feasible pickup and delivery plan a complex one. In addition, these pickups and deliveries need to be synchronised with SL departures in the PDPTW-SL. Thus the problem we consider in this study is NP-hard and requires efficient approaches to be solved. For this reason, and considering the stochastic aspects that make such problems more difficult to handle, one can observe that an SAA approach combined with an ALNS-based heuristic is the mainly used method in the literature for solving similar pickup and deliveries problems (see Li et al. 2016b; Ghilas, Demir, and Van Woensel 2016b). This is because this approach is suitable for testing a wide range of scenarios in a stochastic context while finding good-quality solutions in feasible computational times.

Another important factor regarding the performance of the algorithm is the size of request time windows. As mentioned in Section 5.1, we generate time windows with 40 time units width. Here we evaluate how sensible our algorithm to tighter time windows. We thus solve different instances with 60 requests where time windows width is reduced to 30 and 20 time units and present their average transportation cost and CPU time in Table 7.

First, we observe that the total transportation cost increases when time windows become tighter. This can be explained by the likelihood of serving requests within their time windows. Indeed, the number of requests that require recourse actions, and thus extra transportation costs, increases when time windows are tightened. Similarly, we observe a very slight increase in the computational times needed by the algorithm when the width of request time windows is reduced to 30 and 20 time units respectively. Moreover, we analyse in the following the impacts of uncertainty under different settings including passengers demand and SL frequency and capacity.

\subsubsection{Analysing uncertainty with different levels of passengers demand}

In the original setting, we generate SAA scenarios assuming that passengers demand follows a discrete triangular distribution with a mean value $b=6$ (Figure 7a). In this section, we analyse different levels of passengers demand by testing the algorithm with different mean values ( $b=2,4,6 \& 8$ respectively) while maintaining the same minimum and maximum demand values $(a=0, c=10)$. As the mean value increases, the probability of having a large passengers demand at each SL departure becomes higher. This reflects a real-life case where passengers demand changes over day hours which can limit the integration of PD robot deliveries into the system. The aim of this analysis is thus to investigate the potential impact of these different levels of passengers demand. This is done by performing 10 runs of the algorithm for each demand level and taking the average (Figure 8).

Results show that the total transportation costs increase as passengers demand becomes higher (Figure 8a). This increase is mainly induced by the recourse actions that are used more frequently. Relatively, the average added costs slightly increase from $3.14 \%$ to $3.39 \%$ when passengers demand level goes from 2 to 8 (Figure $8 b$ ). These observations are important for 


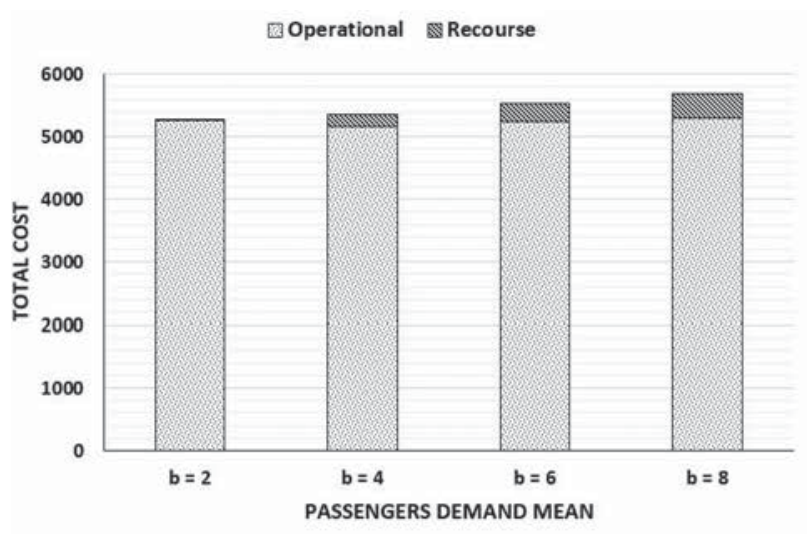

(a)

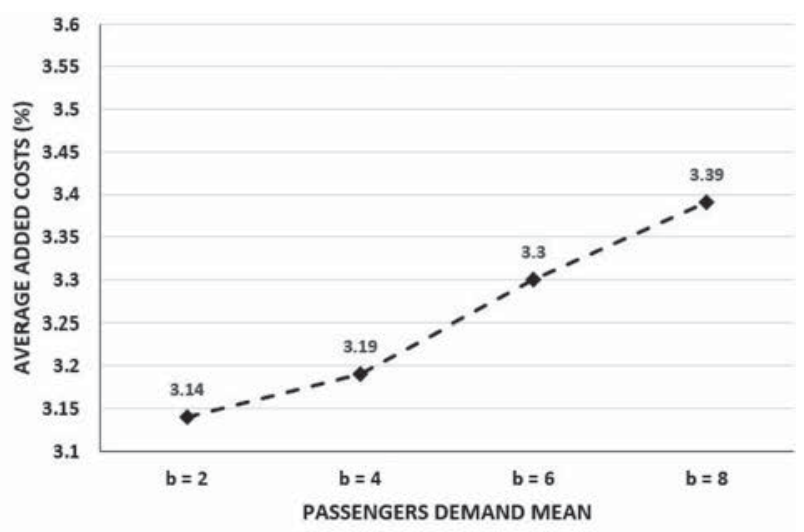

(b)

Figure 8. Passengers demand analysis: (a) average total costs and (b) average added costs.

two main reasons. First, increasing transportation costs indicate that allowing PD robots to be transported with passengers through SLs might not always be efficient when passengers demand is high (e.g. morning and evening peak hours). In other words, this combination can prove most efficient during day hours when the probability of having free places in SLs is bigger. Second, the use of the outsourcing delivery service (action\#3) will increase in peak hours as the subsequent service might also be fully charged with passengers. This means that road traffic can increase as more vehicles are circulating in the system to deliver freight requests that could not be transported using SL service. However, SL combined service still yields many benefits compared to existing freight delivery services, but these benefits can be maximised in off-peak hours.

Furthermore, we compare the proposed integrated system with two different system settings using the varying passengers demand levels. In the first case, we consider that passengers and PD robots are transported in different compartments inside SL shuttles. In other words, shuttles' capacity is divided into two separated spaces: passengers-only and freight-only. In this separated case, the number of places that passengers or PD robots can take in shuttles is fixed and does not depend on passengers demand at each SL departure (e.g. if shuttle capacity is 10 places, 7 of them are reserved for passengers and 3 for PD robots). For testing these settings, we extend the MIP formulation (i.e. first-stage) by adding the capacity constraint of the second-stage (21) and solve it as a deterministic model (i.e. no uncertainty need to be considered as passengers and PD robots are separated). In the second case, we consider a pure-freight system where freight requests are served using a fleet of vehicles without the use of SL services. Each request is thus transported directly from its origin to its destination using a delivery vehicle (this corresponds to using the outsourcing service when applying recourse action\#3).

Results indicate that our integrated system outperforms the pure-freight system in terms of the total transportation with $18.2 \%$ average cost saving (Figure 9). Results also show that these savings decrease when passengers demand becomes higher (from $21.1 \%$ when $b=2$ to $14.8 \%$ when $b=8$ ). This slight decrease is due to the transportation costs added when some requests need to be outsourced due to increasing passengers demand in the integrated system. However, the considerable saving ratio demonstrates the benefits of using the integrated SL service. On the other hand, the integrated system provides a good performance compared to the case where passengers and PD robots are separated (Figure 9). More precisely, this can be observed when passengers demand levels are low (e.g. $b=2$ and $b=4$ ). The system becomes less efficient when demand levels increase while the separated system is not affected. This observation also highlights that integrating passengers and PD robots to the same compartment is less desired at peak-hours, and that its potential is maximised at off-peak hours. From passengers point of view, the integrated system can accommodate more passengers than the separated system where some places are reserved for PD robots. This means that passengers can take full shuttle capacity in the integrated system if necessary, while the separated system can lead to many passengers waiting at stations when their demand's level is high. Our integrated system thus provides a sustainable and flexible solution by allowing freight deliveries only when there is available capacity and without affecting the service provided to passengers.

\subsubsection{Analysing uncertainty with different $S L$ frequencies}

As aforementioned, we consider the SL departure frequency to be 30 time units. We investigate in this section the impact of SL frequency on the total transportation cost. For this purpose, we run the algorithm with SL frequency of 20, 30, 40, 50 and 80 time units and we take the average of 10 runs of the algorithm for each SL frequency (Figure 10). 


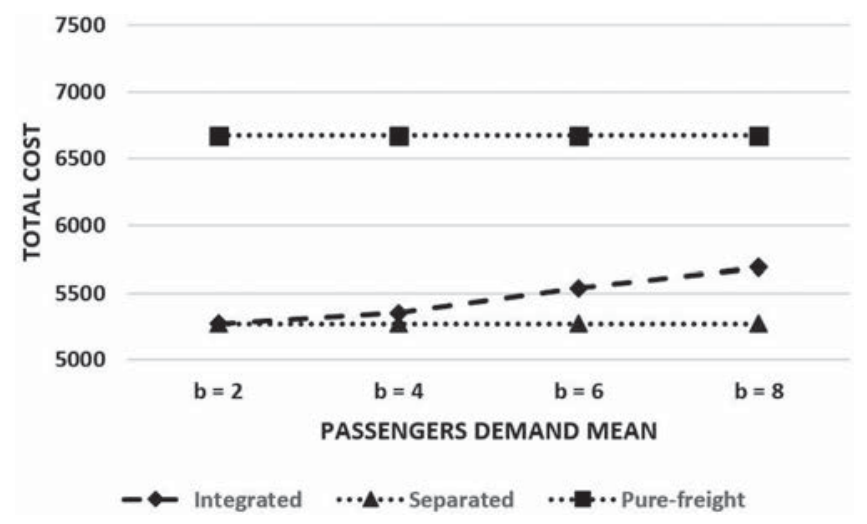

Figure 9. Comparing integrated, separated and pure-freight systems.

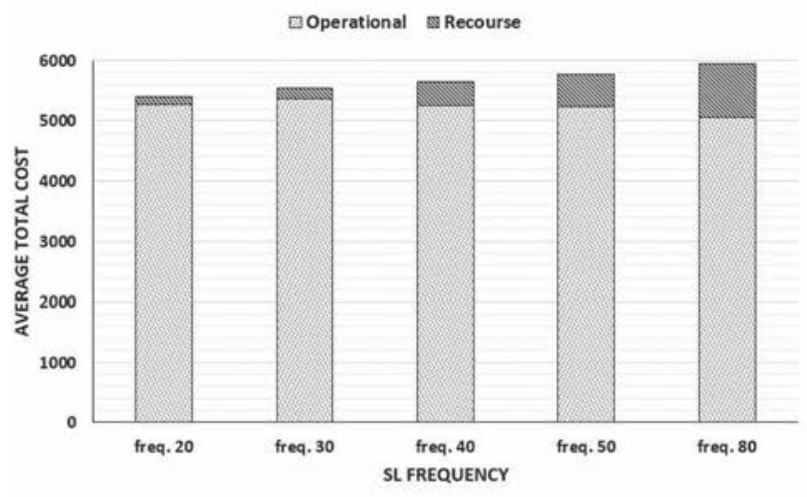

(a)

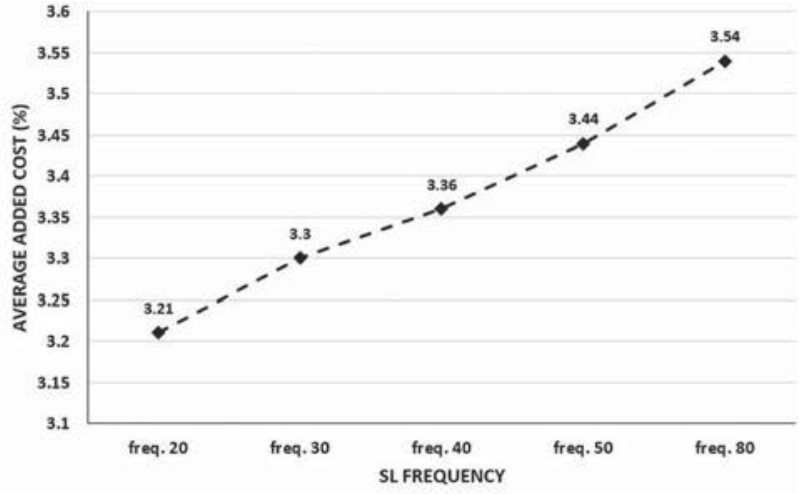

(b)

Figure 10. SL frequency analysis: (a) average total costs and (b) average added costs.

We observe that the total transportation costs increase as SL departures become less frequent. This can be explained by the fact that less frequent SL departures lead to more PD robots waiting at each transfer node which means a higher possibility of having SL capacity violations (Figure 10a). On the other hand, with a more frequent SL service (e.g. 20 time units), the total costs decrease as less recourse actions are needed. This can also be observed by looking at the average added costs with different SL frequencies (Figure 10b). While increasing the SL frequency to 20 time units can reduce the added costs (up to $0.09 \%$ ) compared to the original ones, decreasing the frequency can yield a slightly increasing added costs (up to $0.03 \%, 0.11 \%$ and $0.21 \%$ for 40,50 and 80 SL frequencies respectively). However, increasing SL frequency might also lead to additional costs for SL operators as more shuttles are circulating in the system (e.g. energy, driver wages, etc.). Although freight transportation costs can be decreased by making SLs more frequent, it might not be profitable for SL operators especially at passengers off-peak times. On the other hand, reducing SL frequency can also lead to many passengers left unserved at SL stations. To conclude, increasing, or decreasing, SL frequency needs to take into account the varying levels of passengers demand.

\subsubsection{Analysing uncertainty with different SL capacities}

As introduced earlier, we assumed that PD robots can take up to three places in shuttles. In this section, we investigate the effect of changing the maximum number of places allowed for PD robots on the total transportation costs. As such, we take the average of ten runs of the algorithm with up to five maximum places (Figure 11).

Looking at the obtained results, we observe that allowing more PD robots at each SL departure has a positive effect in terms of the total transportation costs and average added costs. This positive effect is justified by a lowering of $0.07 \%$ and $0.1 \%$ on the added costs when up to 4 or 5 PD robots are allowed at each SL departure. This means that with an extra capacity for PD robots, stochastic solutions become cheaper and less capacity violations can be encountered. However, this 


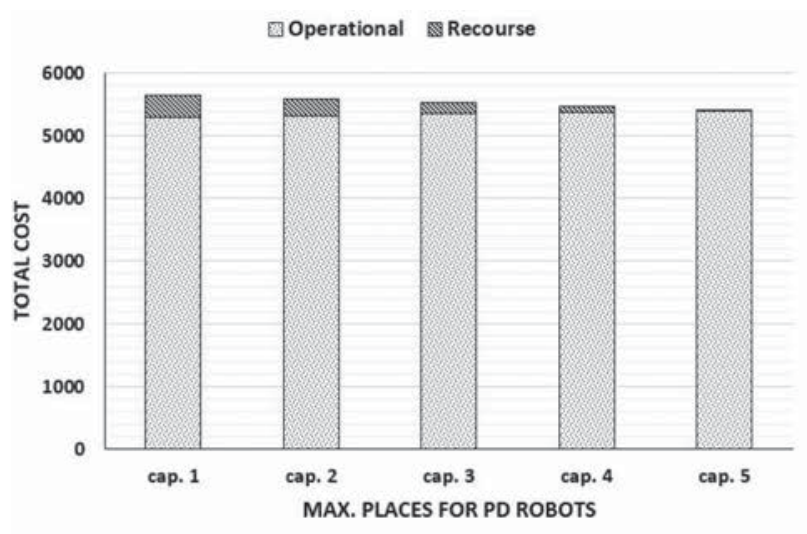

(a)

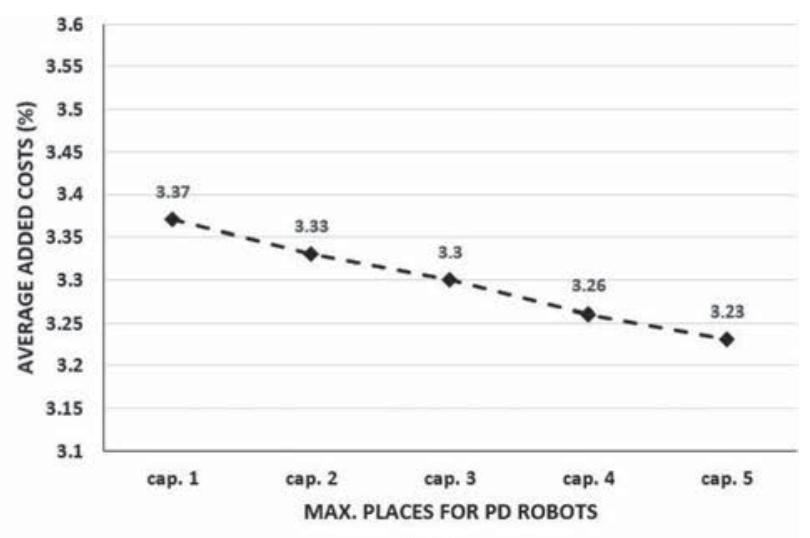

(b)

Figure 11. SL capacity analysis: (a) average total costs and (b) average added costs.

might also have a negative effect on the number of PD robots that have to get off at an intermediate transfer node where passengers demand is high leading to many waiting PD robots at that node.

\section{Conclusion}

In this paper, a transportation service that combines passenger and freight flows has been studied. The associated optimization problem has been formulated as a pickup and delivery problem with time windows, scheduled lines (PDPTW-SL) and stochastic passengers demand. An MIP formulation along with ALNS-based heuristic approach have been introduced. For dealing with uncertainty, a sample average approximation method and a recourse algorithm have been developed. An extensive computational study to evaluate the performance of the proposed approaches and their different components has been presented.

Results of testing instance with up to 60 freight requests showed that the proposed heuristic approach can return solutions that are within $0.6 \%$ of the optimal solutions. The analysis also revealed that an average of $3.3 \%$ extra costs can be observed when stochastic passengers demand is realised. These additional costs reflect the effect of uncertainty on the total transportation costs. Analysing the impact of different SL frequencies and capacities, the results demonstrated the positive effect of increasing the frequency of SL departures and the maximum capacity for PD robots on the system. Finally, our results also showed that the proposed system can achieve an average of $18.2 \%$ cost saving compared to a pure-freight system, and can provide a comparable performance to a system where passengers and freight are separated. This means that such integration represents an opportunity towards more sustainable city logistics.

Since we build our analysis on a set of assumptions that simplify the problem, there are still a number of challenges facing the deployment of such integrated transportation system. Here we outline some directions for future research: (1) we assumed in this paper that each PD robot can only serve one freight request at a time due to the complexity of the considered problem. A more realistic setting would be to allow multiple request pickup and delivery per PD robot trip. This gives rise to the challenge of coupling, or synchronising, both pickup and delivery routing problems as the same PD robot performs them. (2) Another interesting direction would be to study the impact of such integrated service on passenger transportation on a daily horizon in which their demand varies during day hours. Finally, (3) since we consider in this paper one source of uncertainty, which is passengers demand, it is also important to look at other sources of uncertainty like travel times. As PD robots are operating in an urban area, many external factors might affect their travel time and speed. We believe that this study helps in a better understanding of the potential deployment of such integrated systems, and thus, promote more research towards studying this emerging trend in city logistics and transportation.

\section{Acknowledgments}

This research work is carried out as part of the ANTHROPOLIS research project at the Technological Research Institute SystemX and is supported by public funding within the scope of the French Program 'Investissements d'Avenir'.

\section{Disclosure statement}

No potential conflict of interest was reported by the author(s). 


\section{Notes}

1. This integrated system was inspired from Toyota new e-Palette concept. This concept considers designing fully automated, battery electric vehicles that are scalable and customizable for a wide range of Mobility as a Service businesses (MaaS). For example, they can transport passengers, deliver packages, or serve as a mobile office or a roaming medical clinic (the first generation of these vehicles are expected to transport athletes during Tokyo 2020 Olympics, see Toyota e-Palette 2020).

2. We consider PD robots to be small and electric ones so that they can be used for first-mile and last-mile parcel deliveries. They need to be recharged regularly and their maximum distance is thus limited by their battery charge.

3. The algorithm can use the second step only (i.e. randomly assigning PD robots to feasible pickup/drop-off transfer nodes) for large instances where selecting direct deliveries requires a considerable computational effort.

\section{ORCID}

Abood Mourad (D) http://orcid.org/0000-0002-6081-8432

\section{References}

Archetti, C., M. Savelsbergh, and M. G. Speranza. 2016. "The Vehicle Routing Problem with Occasional Drivers.” European Journal of Operational Research 254 (2): 472-480. doi:10.1016/j.ejor.2016.03.049

Arslan, A., N. Agatz, L. G. Kroon, and R. A. Zuidwijk. 2016. "Crowdsourced Delivery - A Pickup and Delivery Problem with Ad-Hoc Drivers.” SSRN Electronic Journal, 1-29. http://www.ssrn.com/abstract = 2726731

Behiri, W., S. Belmokhtar-Berraf, and C. Chu. 2018. "Urban Freight Transport Using Passenger Rail Network: Scientific Issues and Quantitative Analysis." Transportation Research Part E 115: 227-245. doi:10.1016/j.tre.2018.05.002

Ben-Daya, M., E. Hassini, and Z. Bahroun. 2019. "Internet of Things and Supply Chain Management: A Literature Review.” International Journal of Production Research 57 (15-16): 4719-4742. doi:10.1080/00207543.2017.1402140

Cochrane, K., S. Saxe, M. J. Roorda, and A. Shalabi. 2017. "Moving Freight on Public Transit: Best Practices, Challenges, and Opportunities." International Journal of Sustainable Transportation 11: 120-132. doi:10.1080/15568318.2016.1197349

Dayarian, I., and M Savelsbergh. 2017. "Crowdshipping and Same-Day Delivery: Employing In-store Customers to Deliver Online Orders." Optimization Online 7: 42-61.

Dolati Neghabadi, P., K. Evrard Samuel, and M. Espinouse. 2019. "Systematic Literature Review on City Logistics: Overview, Classification and Analysis." International Journal of Production Research 57 (3): 865-887. doi:10.1080/00207543.2018.1489153

Fatnassi, E., J. Chaouachi, and W. Klibi. 2015. "Planning and Operating a Shared Goods and Passengers On-demand Rapid Transit System for Sustainable City-Logistics.” Transportation Research Part B: Methodological 81: 440-460. doi:10.1016/j.trb.2015.07.016

Ghilas, V., J. Cordeau, and E. Demir. 2016. "The Pickup and Delivery Problem with Time Windows and Scheduled Lines: Models and Algorithms.” Transportation Science, 1-30. doi:10.1080/03155986.2016.1166793

Ghilas, V., E. Demir, and T. Van Woensel. 2016a. "An Adaptive Large Neighborhood Search Heuristic for the Pickup and Delivery Problem with Time Windows and Scheduled Lines." Computers and Operations Research 72: 12-30. doi:10.1016/j.cor.2016.01.018

Ghilas, V., E. Demir, and T. Van Woensel. 2016b. "A Scenario-Based Planning for the Pickup and Delivery Problem with Time Windows, Scheduled Lines and Stochastic Demands." Transportation Research Part B: Methodological 91: 34-51. doi:10.1016/j.trb.2016.04.015

Huang, S., A. Potter, and D. Eyers. 2020. "Social Media in Operations and Supply Chain Management: State-of-the-art and Research Directions." International Journal of Production Research 58 (6): 1893-1925. doi:10.1080/00207543.2019.1702228

Jamshidi, A., F. Jamshidi, D. Ait-Kadi, and A. Ramudhin. 2019. "A Review of Priority Criteria and Decision-Making Methods Applied in Selection of Sustainable City Logistics Initiatives and Collaboration Partners.” International Journal of Production Research 57 (15-16): 5175-5193. doi:10.1080/00207543.2018.1540892

Kafle, N., B. Zou, and J. Lin. 2017. "Design and Modeling of a Crowdsource-Enabled System for Urban Parcel Relay and Delivery." Transportation Research Part B: Methodological 99: 62-82. http://linkinghub.elsevier.com/retrieve/pii/S019126151630265X

Li, B., D. Krushinsky, H. A. Reijers, and T. Van Woensel. 2014. "The Share-a-Ride Problem: People and Parcels Sharing Taxis.” European Journal of Operational Research 238 (1): 31-40. doi:10.1016/j.ejor.2014.03.003

Li, B., D. Krushinsky, T. Van Woensel, and H. A. Reijers. 2016a. "An Adaptive Large Neighborhood Search Heuristic for the Share-a-Ride Problem." Computers and Operations Research 66: 170-180. doi:10.1016/j.cor.2015.08.008

Li, B., D. Krushinsky, T. Van Woensel, and H. A. Reijers. 2016b. "The Share-a-Ride Problem with Stochastic Travel Times and Stochastic Delivery Locations.” Transportation Research Part C: Emerging Technologies 67: 95-108. doi:10.1016/j.trc.2016.01.014

Masson, R., A. Trentini, F. Lehuédé, N. Malhéné, O. Péton, and H. Tlahig. 2017. "Optimization of a City Logistics Transportation System with Mixed Passengers and Goods." EURO Journal on Transportation and Logistics 6 (1): 81-109. http://link.springer.com/10.1007/s13676-015-0085-5

Mourad, A., J. Puchinger, and C. Chu. 2019. “A Survey of Models and Algorithms for Optimizing Shared Mobility.” Transportation Research Part B: Methodological 123: 323-346. https://linkinghub.elsevier.com/retrieve/pii/S0191261518304776

Ozturk, O., and J. Patrick. 2018. "An Optimization Model for Freight Transport Using Urban Rail Transit." European Journal of Operational Research 267: 1110-1121. doi:10.1016/j.ejor.2017.12.010

Pathak, D. K., L. S. Thakur, and S. Rahman. 2019. "Performance Evaluation Framework for Sustainable Freight Transportation Systems." International Journal of Production Research 57 (19): 6202-6222. doi:10.1080/00207543.2019.1602741 
Ritzinger, U., J. Puchinger, and R. F. Hartl. 2016. "A Survey on Dynamic and Stochastic Vehicle Routing Problems.” International Journal of Production Research 54 (1): 215-231. doi:10.1080/00207543.2015.1043403

Rossit, D. A., F. Tohmé, and M. Frutos. 2019. “Industry 4.0: Smart Scheduling." International Journal of Production Research 57 (12): 3802-3813. doi:10.1080/00207543.2018.1504248

Savelsbergh, M., and T. Van Woensel. 2016. "50th Anniversary Invited Article - City Logistics: Challenges and Opportunities." Transportation Science 50 (2): 579-590. http://pubsonline.informs.org/doi/10.1287/trsc.2016.0675

Steadieseifi, M., N. P. Dellaert, W. Nuijten, T. Van Woensel, and R. Raoufi. 2014. "Multimodal Freight Transportation Planning: A Literature Review." European Journal of Operational Research 233 (1): 1-15. doi:10.1016/j.ejor.2013.06.055

Tan, X., and J Huang. 2019. "On Computational Complexity of Pickup-and-Delivery Problems with Precedence Constraints or Time Windows." Proceedings of the Twenty-Eighth International Joint Conference on Artificial Intelligence, 5635-5643. https://www.ijcai.org/proceedings/2019/782.

Trentini, A., R. Masson, F. Lehuédé, and N. Malhéné. 2015. "Optimization of a City Logistics Transportation System with Mixed Passengers and Goods." EURO Journal on Transportation and Logistics, 1-23. http://hal.archives-ouvertes.fr/hal-00859078

Verweij, B., S. Ahmed, A. J. Kleywegt, G. Nemhauser, and A. Shapiro. 2003. "The Sample Average Approximation Method Applied to Stochastic Routing Problems: A Computational Study.” Computational Optimization and Applications 24 (1): $289-333$. doi:10.1023/A:1021814225969

Wang, Y., D. Zhang, Q. Liu, F. Shen, and L. H. Lee. 2016. "Towards Enhancing the Last-Mile Delivery: An Effective CrowdTasking Model with Scalable Solutions.” Transportation Research Part E: Logistics and Transportation Review 93: $279-293$. doi:10.1016/j.tre.2016.06.002

\section{Appendix 1}

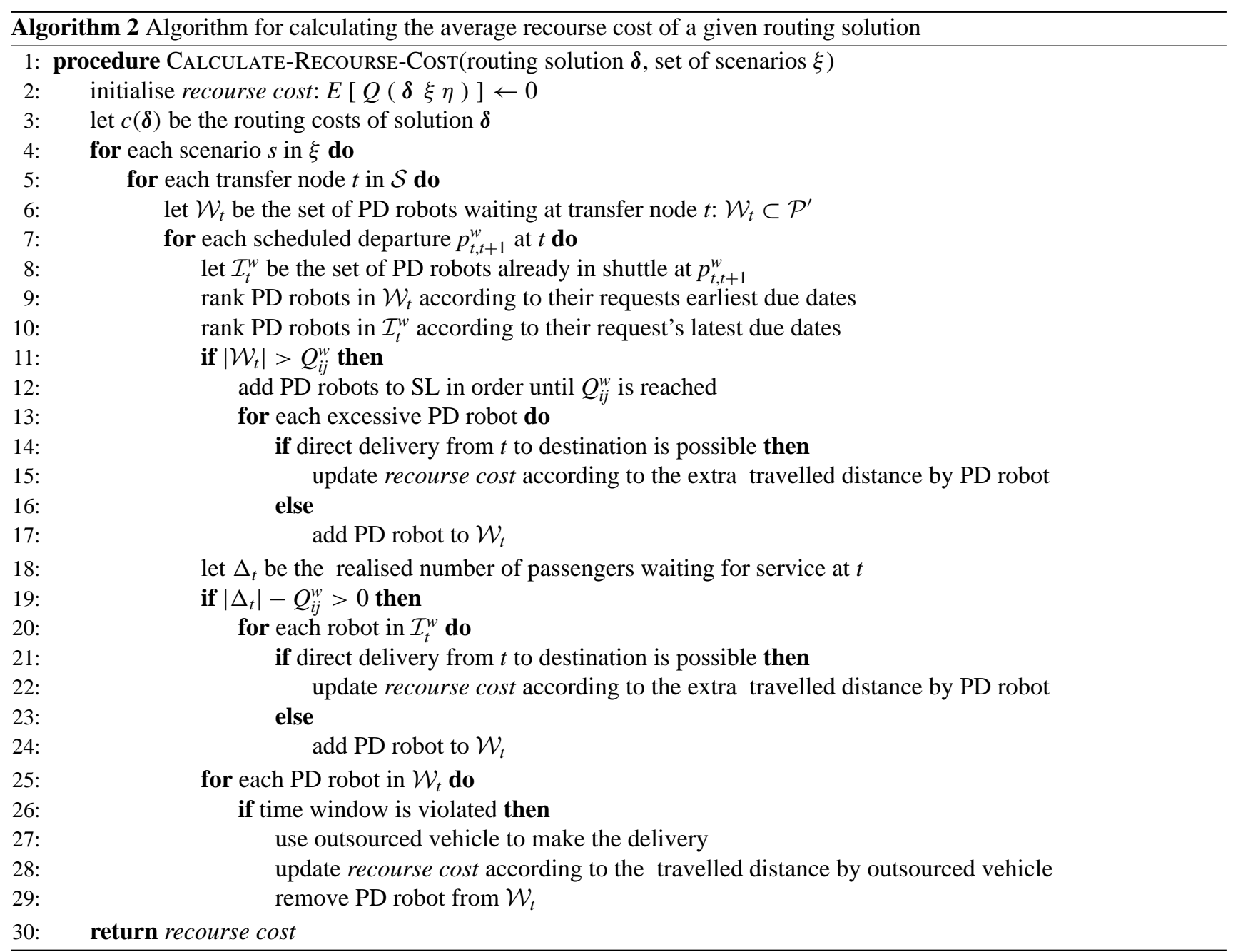




\section{Appendix 2}

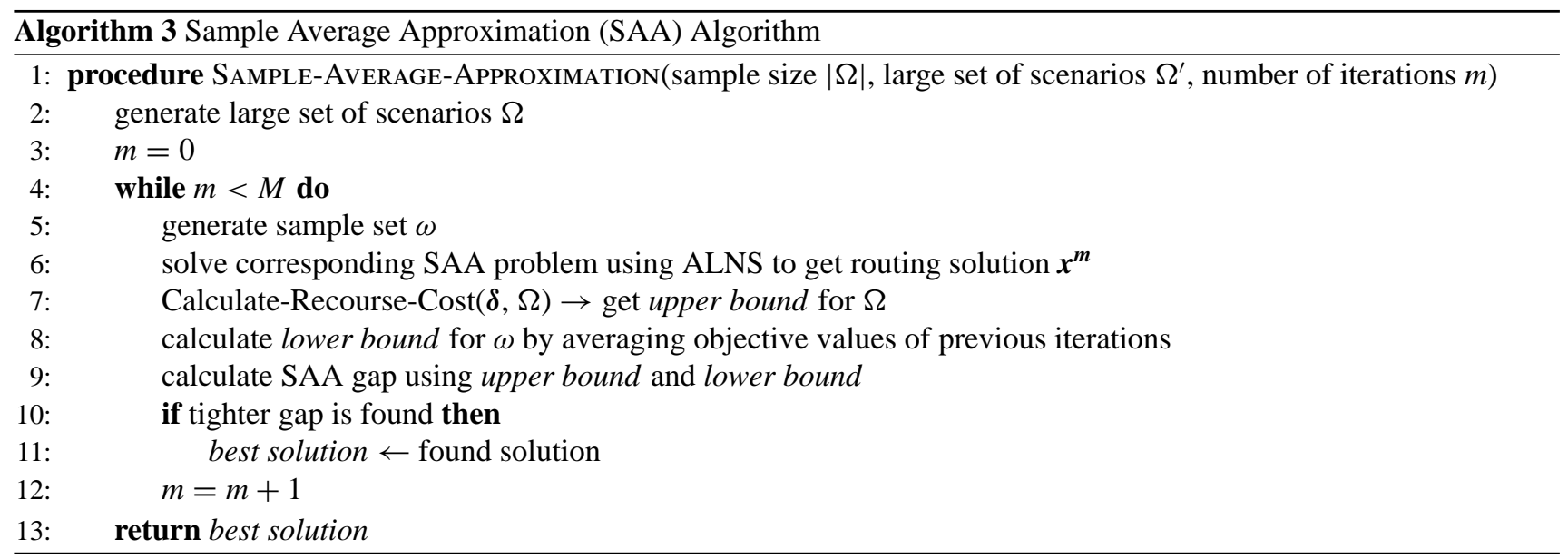

